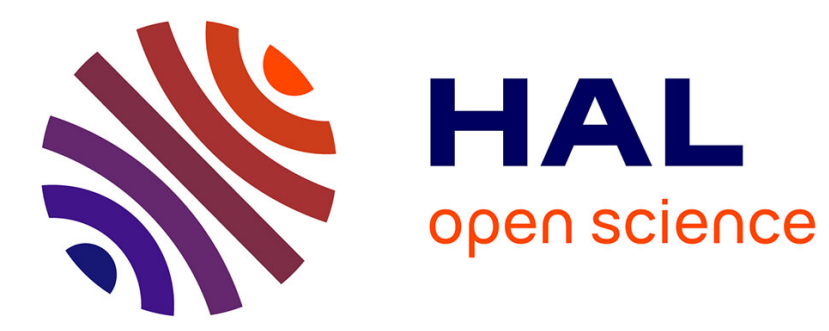

\title{
Experimental characterization of tetrahydrofuran low-temperature oxidation products including ketohydroperoxides and highly oxygenated molecules (HOMs)
}

\author{
Nesrine Belhadj, Roland Benoit, Philippe Dagaut, Maxence Lailliau
}

\section{To cite this version:}

Nesrine Belhadj, Roland Benoit, Philippe Dagaut, Maxence Lailliau. Experimental characterization of tetrahydrofuran low-temperature oxidation products including ketohydroperoxides and highly oxygenated molecules (HOMs). Energy \& Fuels, 2021, 35 (9), pp.7242-7252. 10.1021/acs.energyfuels.0c03291 . hal-03221818

\section{HAL Id: hal-03221818 \\ https://hal.science/hal-03221818}

Submitted on 10 May 2021

HAL is a multi-disciplinary open access archive for the deposit and dissemination of scientific research documents, whether they are published or not. The documents may come from teaching and research institutions in France or abroad, or from public or private research centers.
L'archive ouverte pluridisciplinaire HAL, est destinée au dépôt et à la diffusion de documents scientifiques de niveau recherche, publiés ou non, émanant des établissements d'enseignement et de recherche français ou étrangers, des laboratoires publics ou privés.

$$
\text { Copyright }
$$




\title{
Experimental characterization of tetrahydrofuran low-temperature oxidation products including ketohydroperoxides and highly oxygenated molecules (HOMs).
}

\author{
Nesrine Belhadj ${ }^{1,2}$, Roland Benoit ${ }^{1}$, Philippe Dagaut ${ }^{1{ }^{*}}$, Maxence Lailliau ${ }^{1,2}$ \\ ${ }^{1}$ Centre National de la Recherche Scientifique, ICARE, Orléans, France \\ ${ }^{2}$ Université d'Orléans, Orléans, France
}

*Corresponding author:

Philippe Dagaut

CNRS-ICARE, Institut de Combustion, Aérothermique, Réactivité et Environnement

1C Avenue de la Recherche Scientifique

45071 Orléans Cedex 2, France

Tel: +33(0)238255466

dagaut@,cnrs-orleans.fr

\begin{abstract}
The oxidation of tetrahydrofuran (THF) was carried out in a continuously jet-stirred tank reactor (JSR) at a total pressure of $10 \mathrm{~atm}$, in fuel-lean conditions (equivalence ratio $=0.5$ ), an initial fuel mole fraction of $5000 \mathrm{ppm}$, at a mean residence time of $2 \mathrm{~s}$, and for temperatures ranging from 550 to $620 \mathrm{~K}$. High-resolution mass spectrometry analyses was used to characterize low-temperature oxidation products of THF. MS analyses were performed using atmospheric pressure chemical ionizations in positive and negative modes. Both flow injection analyses and ultra-high-pressure liquid chromatography-MS/MS allowed characterizing a large set of chemicals including hydroperoxides and diols $\left(\mathrm{C}_{4} \mathrm{H}_{8} \mathrm{O}_{3}\right)$, ketohydroperoxides $\left(\mathrm{C}_{4} \mathrm{H}_{6} \mathrm{O}_{4}\right)$, and more oxygenated molecules (up to $\mathrm{C}_{4} \mathrm{H}_{8} \mathrm{O}_{7}$ ) resulting from up to three oxygen molecules addition of on $\alpha$ and $\beta$ THF radicals. The existence of $\mathrm{OH}$ or $-\mathrm{OOH}$ groups in the products was confirmed by hydrogen-deuterium exchange using $\mathrm{D}_{2} \mathrm{O}$. We detected 24 products with molecular weight of 40-168, not reported in previous studies. Simulations using the latest THF oxidation chemical kinetic reaction mechanism available from the literature were compared to the present measurements of ketohydroperoxides and other products of THF cool flame. The kinetic scheme represented well the present qualitative data.
\end{abstract}

Keywords: tetrahydrofuran, oxidation, jet-stirred reactor, cool flame, ketohydroperoxides, HOMs 


\section{INTRODUCTION}

It is well-known that cyclic ethers (oxiranes, oxetanes, tetrahydrofurans, and tetrahydropyrans) are important products of liquid fuels cool flames, as observed in a variety of environments, e.g. in research engines ${ }^{1-4}$ and in laboratory experiments. ${ }^{5-8}$ Tetrahydrofurans and oxetanes are generally the most abundant cyclic ethers formed in cool flames. ${ }^{6-7,9-10}$ Following reactions of these products may affect the overall reactivity of the system. Furthermore, there is growing interest in using furanics as bioderived ground transportation fuels. ${ }^{11}$ Thus more information is needed for assessing their kinetics of oxidation and the production of fuel-specific pollutants.

To date, in contrast with linear light ethers, e.g. dimethyl ether and diethyl ether, little information is available for the oxidation reaction mechanisms of cyclic ethers ${ }^{4,12-15}$ and in particular for THF which has a cetane number $\sim 22 .{ }^{16}$ Indeed, in the literature, one can find few studies on THF low- to intermediate-temperature oxidation, e.g. oxidation in a static reactor at $493 \mathrm{~K},{ }^{17}$ high-pressure studies in rapid compression machine and shock-tube, ${ }^{18-21}$ rapid compression machine (RCM) and JSR studies, ${ }^{15,} 18,22$ and Ignition Quality Tester measurements. ${ }^{23}$ More recently, Hansen et al. ${ }^{24}$ presented a detailed experimental and theoretical study of the formation of KHPs in a JSR under cool flame conditions. A comparison with modeling using Fenard et al. model ${ }^{22}$ indicated discrepancies in terms of relative importance of KHP isomers. Products have been measured by synchrotronbased technique (vacuum ultra-violet photoionization-molecular beam-time of flight mass spectrometry) for the fuel-lean (equivalence ratio $=0.3$ ) oxidation of $10,000 \mathrm{ppm}$ of THF at $\sim 1 \mathrm{~atm}$ and a residence time of $2 \mathrm{~s}$. Several KHPs have been detected, but no products resulting from third or higher molecular oxygen addition on THF radicals have been reported whereas they are expected to be formed, based on recent works involving a range of fuels. ${ }^{25-28}$ While the production of highly oxygenated molecules in combustion is commonly overlooked, but could promote ignition according to previous chemical kinetic modeling, ${ }^{29}$ they are considered being very important intermediates participating to the formation of secondary organic aerosols (SOA) in the troposphere. ${ }^{30}$ Previous chromatographic separation of KHPs deriving from the oxidation of large hydrocarbons have been detected by mass spectrometry and ultraviolet absorption, ${ }^{31-39}$ but these earlier investigations were technically limited. With the recent development of analytical techniques, e.g., synchrotron-based mass spectrometry, ${ }^{25,} 40-41$ ultra-high-pressure liquid chromatography (UHPLC) coupled to Orbitrap ${ }^{\circledR}$, it becomes possible to better characterize cool flame products. ${ }^{26-27,42}$

As part of continuing efforts in this laboratory to better understand the chemical kinetics of fuels combustion, a series of experiments has been carried out recently for the oxidation of several ethers. ${ }^{26-27}$ In this paper we extend that work by presenting results of kinetic experiments completed to identify the products of THF cool flame in a JSR. Among the products detected, we paid particular attention to the detection of ketohydroperoxide isomers since discrepancies between kinetic modeling and measurements have been reported recently ${ }^{24}$. Chemical kinetic modeling was performed to extend the validation of a published $\operatorname{model}^{22}$.

\section{EXPERIMENTAL}

\subsection{Oxidation Experiments}

In the present experimental study we used a jet-stirred reactor setup introduced previously ${ }^{43-44}$ and used in former studies. ${ }^{45-48}$ The fused silica JSR ( $35 \mathrm{~cm}^{3}, 4$ nozzles of $1 \mathrm{~mm}$ I.D. for stirring), installed inside a regulated oven (ca. $1.5 \mathrm{~kW}$ ) which maintains the temperature of the JSR at desired working temperatures. This assembly is 
located inside a pressure-resistant stainless-steel housing. The oven is thermally insulated by ceramic wool. The JSR working temperature was varied stepwise while flow rates were adjusted to keep mean residence time constant under all the investigated conditions. As in earlier studies, ${ }^{49}$ THF ( $>99.9 \%$ pure from Sigma Aldrich, CAS Number 109-99-9) was atomized by a flow of nitrogen and vaporized in a heated chamber. THF and oxygen were delivered separately to the JSR to avoid fuel oxidation before entrance into the reactor. Nitrogen and oxygen flow rates were regulated by mass flow controllers. THF was pumped by a Shimadzu LC10-AD-VP HPLC pump. Thanks to the use of an online degasser (Shimadzu DGU-20-A3) the pump could deliver a steady flow rate of THF. Table 1 summarizes the present experimental conditions.

Table 1. Experimental conditions

\begin{tabular}{|l|l|}
\hline Equivalence ratio $(\varphi)$ & 0.5 \\
\hline Pressure & $10 \mathrm{~atm}$ \\
\hline Initial concentrations & $0.5 \%$ of fuel, $2.75 \% \mathrm{O}_{2}, 96.75 \% \mathrm{~N}_{2}$ \\
\hline Residence time & $2 \mathrm{~s}$ \\
\hline Temperature & $550-620 \mathrm{~K}$ \\
\hline
\end{tabular}

A Pt-Pt/Rh-10\% thermocouple $(0.1 \mathrm{~mm}$ wires located inside a thin-wall fused-silica tube $)$ was used to check thermal homogeneity along the vertical axis of the JSR. It was found good (gradients of $<1 \mathrm{~K} / \mathrm{cm}$ ). A lowpressure sonic probe (fused-silica) was used to freeze reactions and collect samples which will be analyzed off line. To characterize THF low-temperature oxidation products, e.g., hydroperoxides, ketohydroperoxides (KHPs), ketodi-hydroperoxides, and other oxygenates, the gaseous samples were bubbled for $75 \mathrm{~min}$ in cooled acetonitrile $\left(0^{\circ} \mathrm{C}, 25 \mathrm{~mL}\right.$ in a $40 \mathrm{~mL}$ amber glass vial). The liquid samples were stored at $-15^{\circ} \mathrm{C}$ for preventing degradation before chemical analyses.

\subsection{Chemical analyses}

Several types of analyses were performed. Firstly, mass spectrometry (MS) analyses with an Orbitrap ${ }^{\circledR}$ QExactive were performed by flow injection (FIA). There, a motored syringe is used to inject continuously the sample in the ionization chamber of the mass spectrometer ${ }^{50}$. The FIA conditions were: flow of 1-3 $\mu \mathrm{L} / \mathrm{min}$ recorded for $1 \mathrm{~min}$. The samples were introduced into the ionization chamber of the Orbitrap ${ }^{\circledR}$ (mass accuracy $<1$ ppm RMS and mass resolution of 140,000). Secondly, ultra-high-pressure liquid chromatography-MS (Vanquish Flex UHPLC from Thermo Scientific) was used. For samples ionization, atmospheric chemical ionization (Ion Max ${ }^{\circledR}$ APCI) operating in positive and negative modes was used. APCI is a soft ionization method widely used in liquid chromatography-MS ${ }^{51}$. Compared to electrospray ionization ESI which uses an electric field and photoionization PI (using UV radiation), in APCI a corona discharge needle is used to produce an electric discharge applied to the solvent aerosol which produces positive or negative radical ions $\left(\mathrm{N}_{2}{ }^{\cdot+}, \mathrm{O}_{2}{ }^{\cdot+}, \mathrm{H}_{2} \mathrm{O}^{\cdot+}, \mathrm{NO}^{\cdot+}\right.$, $\mathrm{O}_{2}{ }^{--}, \mathrm{O}^{+}, \mathrm{O}^{*}, \mathrm{NO}_{2}{ }^{-*}, \mathrm{O}_{3}^{-*}$ ), which transfer their charges to the sample molecules and ionize them. APCI is used for ionizing polar and relatively less polar compounds $(<1500 \mathrm{Da})$ including organic pollutants, pesticides, 
pharmaceutical products, biological chemicals, and drug metabolites. APCI has also been used to ionize non-polar and high molecular weight chemicals, e.g., hydrocarbons, polycyclic aromatic hydrocarbons (PAHs), and polymers. ${ }^{52-53}$

For mass calibrations, we used the commercial Pierce ${ }^{\mathrm{TM}}$ ESI $(+)$ and (-) calibration mixtures (Thermo Scientific) in FIA mode and HESI.

Most of the chromatographic analyses were performed with a $\mathrm{C}_{18}$ analytical column (Phenomenex Luna, $1.6 \mu \mathrm{m}, 100 \AA, 100 \times 2.1 \mathrm{~mm}, 40^{\circ} \mathrm{C}$ ) in reverse-phase mode. Additional HPLC-MS analyses were performed with two other types of columns: Hypercarb PGC (Thermo Scientific, $5 \mu \mathrm{m}, 150 \times 2.1 \mathrm{~mm}, 40^{\circ} \mathrm{C}$ ) and Ascentis Si (Supelco, $5 \mu \mathrm{m}, 250 \times 2.1 \mathrm{~mm}, 40^{\circ} \mathrm{C}$ ). The analytical conditions are given in the Supporting information (Table $\mathrm{S} 1)$.

Besides mass calibrations, mass spectra of standards and retention times were used to identify products by UHPLC-MS/MS ( $\gamma$-Butyrolactone, THF-3-one, cyclopropane carboxaldehyde, 3,4-epoxy tetrahydrofuran, 2,3DHF, 2,5-DHF, succinic acid, and furan). UHPLC and HPLC analyses were performed using optimized elution solvent and gradients (Supporting information, Table S2).

To identify THF oxidation products, MS-MS analyses were performed at several collision cell energies (10, 30,50 , and $70 \mathrm{eV}$ ). The fast $\mathrm{OH} / \mathrm{OD}$ exchange used in previous studies ${ }^{25-27}$ was used to confirm the presence of hydroxy and hydroperoxy groups in THF oxidation products. This is a technique widely used in combination with MS, as described in details in a recent review article. ${ }^{54}$ Here, we introduced $60 \mu \mathrm{L}$ of $\mathrm{D}_{2} \mathrm{O}(99.98 \% \mathrm{D}$, SigmaAldrich) into $200 \mu \mathrm{L}$ of sample (reaction time $25 \mathrm{~min}$ ). with the $70 \%$ evaporated sample collected at $600 \mathrm{~K}$ and $10 \mathrm{~atm}$. The obtained solution was analyzed by FIA Orbitrap ${ }^{\circledR}$ and APCI positive and negative modes (Supporting information, Figure S1). The signal profiles reported in Section 4 have an estimated uncertainty of $40 \%$. Uncertainties include typical $10-15 \%$ uncertainties in JSR experiments, solubility of species in acetonitrile, possible loss of species with vaporizer temperature and capillary temperature of APCI source around $400 \mathrm{~K}$ and $600 \mathrm{~K}$, respectively, instabilities of MS signal during analyses.

\section{KINETIC MODELING}

For the computations we used the PSR software ${ }^{55}$ from the Chemkin II package ${ }^{56}$. A previously published kinetic reaction mechanism for THF oxidation, ${ }^{22}$ validated over an extensive range of conditions, was used here. It includes both low- and high-temperature oxidation routes, but is limited to the second addition of molecular oxygen on THF radicals, yielding ketohydroperoxides. According to the present simulations, under the present conditions, THF mainly reacts by $\mathrm{H}$-atom abstraction with the hydroxyl radical, yielding two fuel's radicals, $\mathrm{R}$, in $\alpha$ - and $\beta$-position to the ether function (Table 2).

Table 2. Chemical structure of fuel's radicals, $\mathrm{ROOH}$, and ketohydroperoxides produced during THF oxidation.

\begin{tabular}{|c|c|}
\hline \multicolumn{2}{|c|}{ Initial radicals produced by H-atom abstraction on THF } \\
\hline$\alpha$-position & $\beta$-position \\
\hline
\end{tabular}




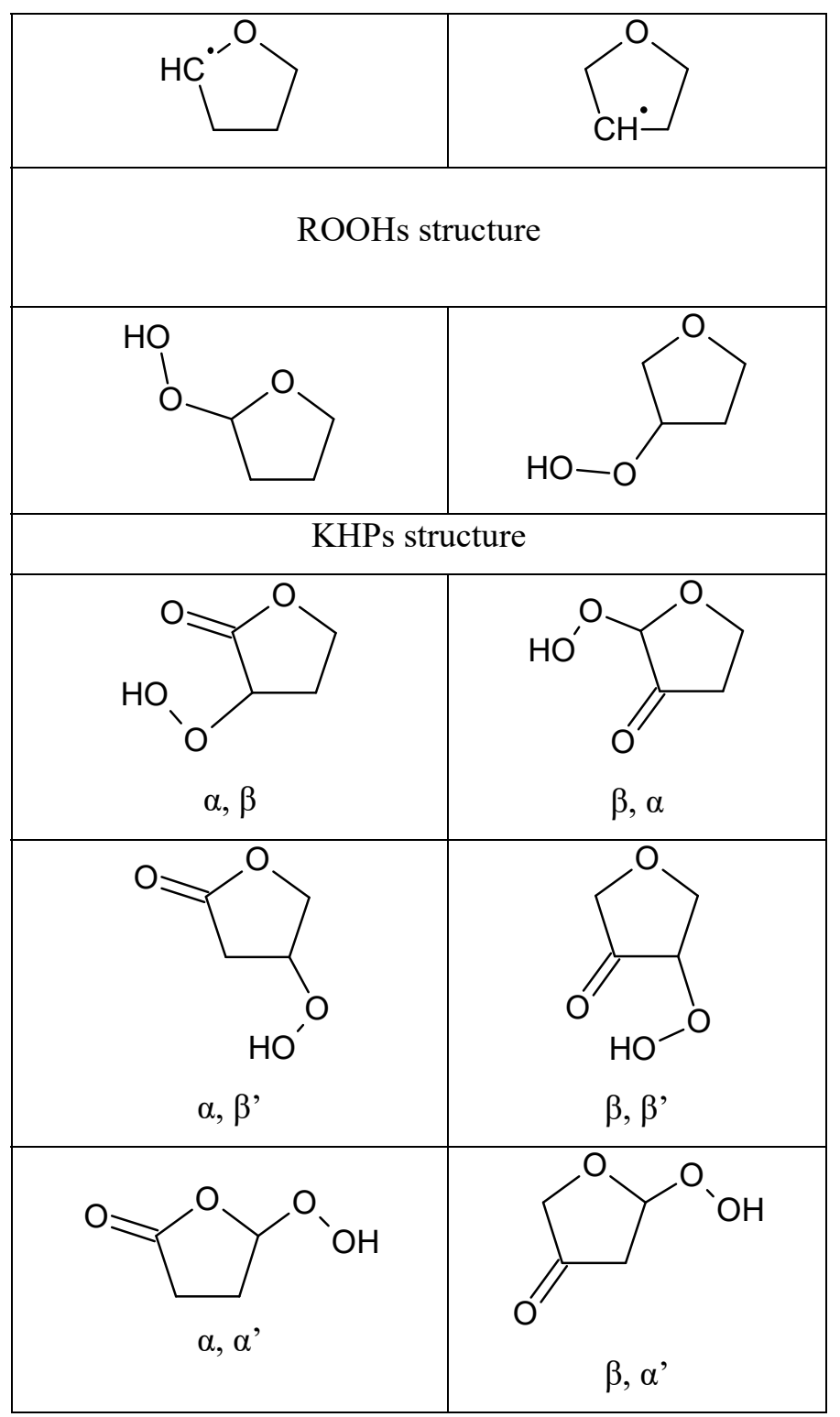

Below ca. $900 \mathrm{~K}$, these two radicals get peroxidized by addition of molecular oxygen. Two hydroperoxides resulting from $\mathrm{H}$-atom abstraction $\left(\mathrm{ROO}+\mathrm{R}^{\prime} \mathrm{H} \leftrightarrows \mathrm{ROOH}+\mathrm{R}^{\prime}\right)$ can be formed. After intramolecular $\mathrm{H}$-atom transfer in fuel's peroxy radicals, yielding an hydroperoxy radical, a second molecular oxygen addition, itself followed by intramolecular H-atom transfer and decomposition, can yield up to 6 ketohydroperoxides (Table 2).

A schematic representation of THF oxidation pathways is given in Figure 1. Presently detected species appear in boxes. 


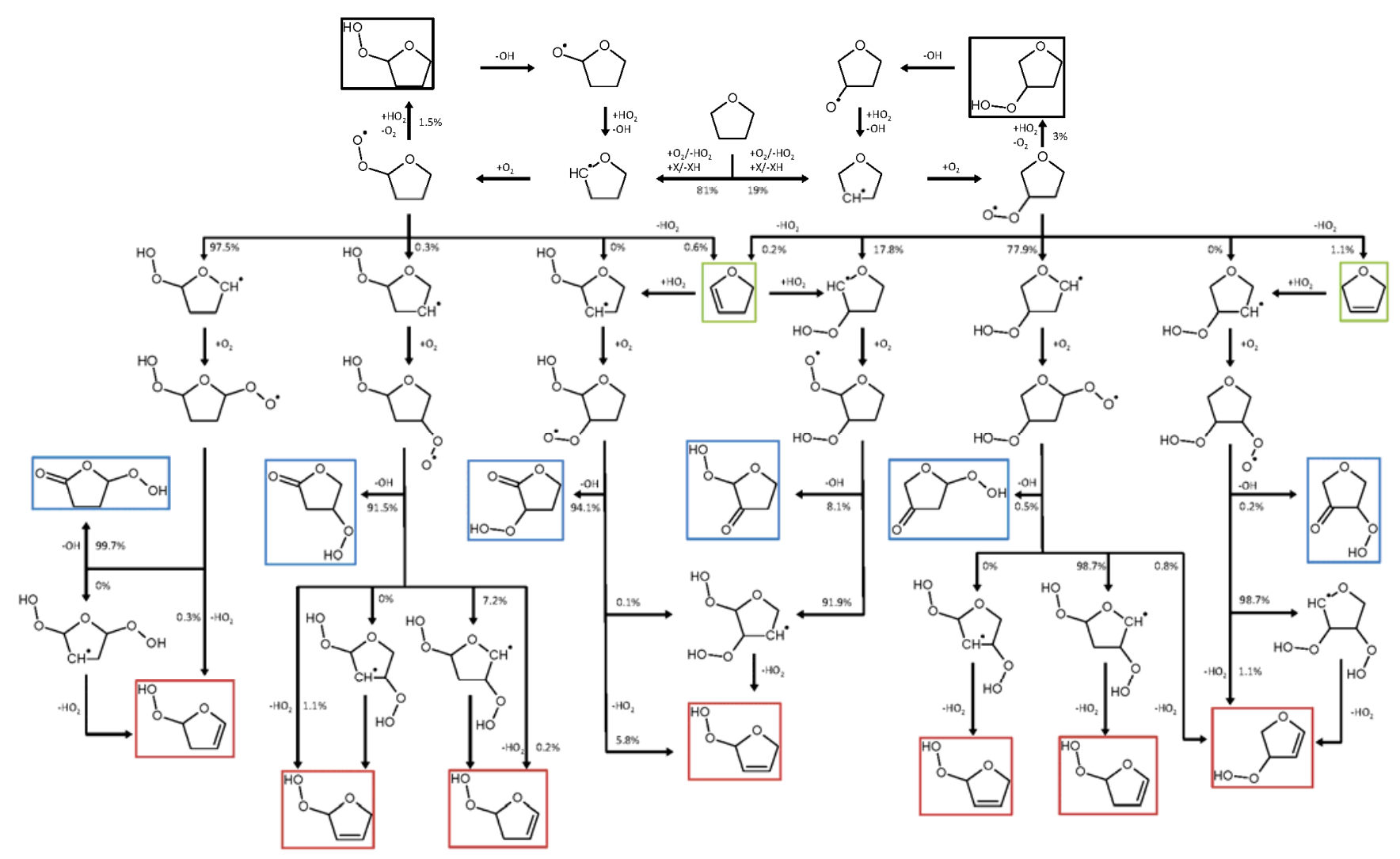

Figure 1. Schematic representation of THF oxidation pathways at $580 \mathrm{~K}$, under the condition of this study (Table 1). The model of Fenard et al..$^{22}$ was used. Fluxes lower than $0.1 \%$ are shown as zero.

\section{RESULTS AND DISCUSSION}

THF oxidation was studied in a JSR at elevated pressure and fuel-lean conditions (Table 1) yielded a large set of low-temperature oxidation products. Products chemical formulae were determined by high-resolution mass spectrometry: $\mathrm{C}_{4} \mathrm{H}_{6} \mathrm{O}_{\mathrm{x}}(\mathrm{x}=0-6), \mathrm{C}_{4} \mathrm{H}_{4} \mathrm{O}_{\mathrm{x}}(\mathrm{x}=1-5), \mathrm{C}_{4} \mathrm{H}_{2} \mathrm{O}_{\mathrm{x}}(\mathrm{x}=2-3), \mathrm{C}_{\mathrm{n}} \mathrm{H}_{2 \mathrm{n}}(\mathrm{n}=2-4), \mathrm{C}_{\mathrm{n}} \mathrm{H}_{2 \mathrm{n}-2}(\mathrm{n}=2-4), \mathrm{C}_{\mathrm{n}} \mathrm{H}_{2 \mathrm{n}} \mathrm{O}(\mathrm{n}=2-$ 4), $\mathrm{C}_{n} \mathrm{H}_{2 n-2} \mathrm{O}(\mathrm{n}=3-4), \mathrm{C}_{\mathrm{n}} \mathrm{H}_{2 n-4} \mathrm{O}(\mathrm{n}=3-4), \mathrm{C}_{\mathrm{n}} \mathrm{H}_{2 \mathrm{n}+2} \mathrm{O}_{2}(\mathrm{n}=2-4), \mathrm{C}_{\mathrm{n}} \mathrm{H}_{2 \mathrm{n}} \mathrm{O}_{2}(\mathrm{n}=2-4), \mathrm{C}_{\mathrm{n}} \mathrm{H}_{2 n-2} \mathrm{O}_{2}(\mathrm{n}=2-4), \mathrm{C}_{n} \mathrm{H}_{2 n-4} \mathrm{O}_{2}(\mathrm{n}=3-$ 4), and $\mathrm{C}_{\mathrm{n}} \mathrm{H}_{2 \mathrm{n}} \mathrm{O}_{3}(\mathrm{n}=2-4)$. Alkenes, dienes, furan, di-hydrofurans, alcohols, 1,4-dioxane, carbonyl compounds, organic hydroperoxides, ketohydroperoxides, diketones, bicyclic ethers, carboxylic acids, and highly-oxygenated products were identified, in agreement with previous studies (Table 3). Indeed, besides ketohydroperoxides, more oxygenated products resulting from multiple addition of molecular oxygen on THF radicals (R) were observed here. The following sequence of reactions describes pathways to such products:

$$
\begin{gathered}
\mathrm{R}+\mathrm{O}_{2} \leftrightarrows \mathrm{RO}_{2} \\
\mathrm{RO}_{2} \leftrightarrows \mathrm{QOOH} \\
\mathrm{QOOH}+\mathrm{O}_{2} \leftrightarrows \mathrm{OOQOOH} \\
\mathrm{OOQOOH} \leftrightarrows \mathrm{HOOQ} \mathrm{OOH} .
\end{gathered}
$$


This last reaction is followed by decomposition of di-hydroperoxy radicals, HOOQ'OOH, yielding the hydroxyl radical and a ketohydroperoxide $\left(\mathrm{C}_{4} \mathrm{H}_{6} \mathrm{O}_{4}\right)$ :

$$
\mathrm{HOOQ}{ }^{\prime} \mathrm{OOH} \rightarrow \mathrm{OH}+\mathrm{HOOQ} \mathrm{O}_{\left(\mathrm{C}_{4} \mathrm{H}_{6} \mathrm{O}_{4}\right)} .
$$

Also, di-hydroperoxy radicals can react with $\mathrm{O}_{2}$ (third addition of molecular oxygen), followed by intramolecular $\mathrm{H}$-atom transfer and decomposition of organic hydroperoxy radicals:

$$
\begin{gathered}
\mathrm{HOOQ}{ }^{\prime} \mathrm{OOH}+\mathrm{O}_{2} \leftrightarrows(\mathrm{HOO})_{2} \mathrm{Q}^{\prime} \mathrm{OO} \\
(\mathrm{HOO})_{2} \mathrm{Q}^{\prime} \mathrm{OO} \leftrightarrows(\mathrm{HOO})_{2} \mathrm{POOH} \\
(\mathrm{HOO})_{2} \mathrm{Q}^{\prime} \mathrm{OO} \rightarrow \mathrm{OH}+(\mathrm{HOO})_{2} \mathrm{P}=\mathrm{O}\left(\mathrm{C}_{4} \mathrm{H}_{6} \mathrm{O}_{6}\right)
\end{gathered}
$$

Oxidation could further continue via a fourth addition of molecular oxygen yielding more oxygenated products:

$$
\begin{gathered}
(\mathrm{HOO})_{2} \mathrm{POOH}+\mathrm{O}_{2} \rightarrow(\mathrm{HOO})_{3} \mathrm{POO} \\
(\mathrm{HOO})_{3} \mathrm{POO} \leftrightarrows(\mathrm{HOO})_{3} \mathrm{P}^{\prime} \mathrm{OOH} \\
(\mathrm{HOO})_{3} \mathrm{P}^{\prime} \mathrm{OOH} \rightarrow \mathrm{OH}+(\mathrm{HOO})_{3} \mathrm{P}^{\prime}=\mathrm{O}\left(\mathrm{C}_{4} \mathrm{H}_{6} \mathrm{O}_{8}\right) .
\end{gathered}
$$

The present study confirms the occurrence of extended oxidation pathways ${ }^{25,57}$ deduced from JSR experiments with synchrotron-based mass spectrometry. ${ }^{25,57}$ In these experiments, several products of the $2^{\text {nd }}$ and $3^{\text {rd }}$ addition of molecular oxygen were detected. More recently, ${ }^{26-27}$ products resulting from addition on fuels radicals of up to 4,4 , and $6 \mathrm{O}_{2}$, respectively, have been observed in experiments similar to those performed here. Observation of the products of the $4^{\text {th }} \mathrm{O}_{2}$ addition on fuel's radicals (keto-tri-hydroperoxide $\mathrm{C}_{4} \mathrm{H}_{6} \mathrm{O}_{8}$ ) was scarce in the present oxidation experiments with THF. However, the products of $2^{\text {nd }}$ and $3^{\text {rd }} \mathrm{O}_{2}$ addition were well detected and are reported in Table 3.

Organic hydroperoxides, $\mathrm{ROOH}$, are minor products formed by $\mathrm{H}$-atom abstraction by peroxy radicals:

$$
\begin{gathered}
\mathrm{RO}_{2}+\mathrm{R}^{\prime} \mathrm{H} \rightarrow \mathrm{ROOH}+\mathrm{R}^{\prime} \\
\mathrm{RO}_{2}+\mathrm{HO}_{2} \rightarrow \mathrm{ROOH}+\mathrm{O}_{2} .
\end{gathered}
$$

Their formation was observed here (Table 3). The variation of the integrated $\mathrm{C}_{4} \mathrm{H}_{9} \mathrm{O}_{3}{ }^{+}$signal obtained by FIA vs. temperature is presented in Figure 2. These qualitative results were compared to simulations (Figure 2) showing consistent variation of their importance with temperature. In the chemical scheme of Fenard et al., the reaction $\mathrm{RO}_{2}+\mathrm{R}$ 'H was not included. Thus, only the reaction with $\mathrm{HO}_{2}$ is important in the simulations. 


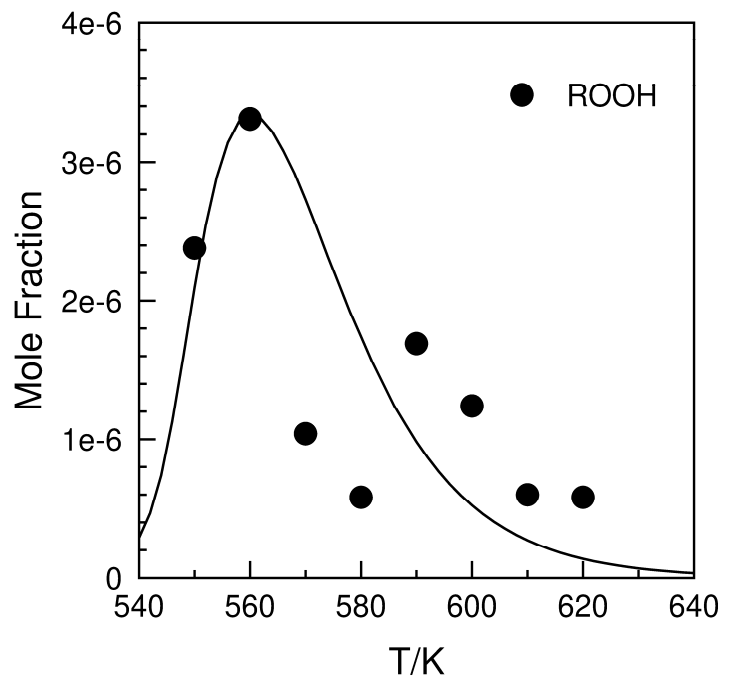

Figure 2. Formation of $\mathrm{C}_{4} \mathrm{H}_{8} \mathrm{O}_{3}$ in a JSR by oxidation of $5000 \mathrm{ppm}$ of THF. The analyses were performed in FIA and APCI $(+)$ mode. The data (dots) represent the signal recorded at $\mathrm{m} / \mathrm{z} 105.0545$, scaled to the maximum computed mole fraction (line).

$\mathrm{C}_{4} \mathrm{H}_{8} \mathrm{O}_{3}$ could also be diols which can result from the decomposition of di-hydroperoxides. ${ }^{36}$ The observation of two H/D exchange (Figure 3) seems to indicate diols were formed in the present experiments (Table 3).
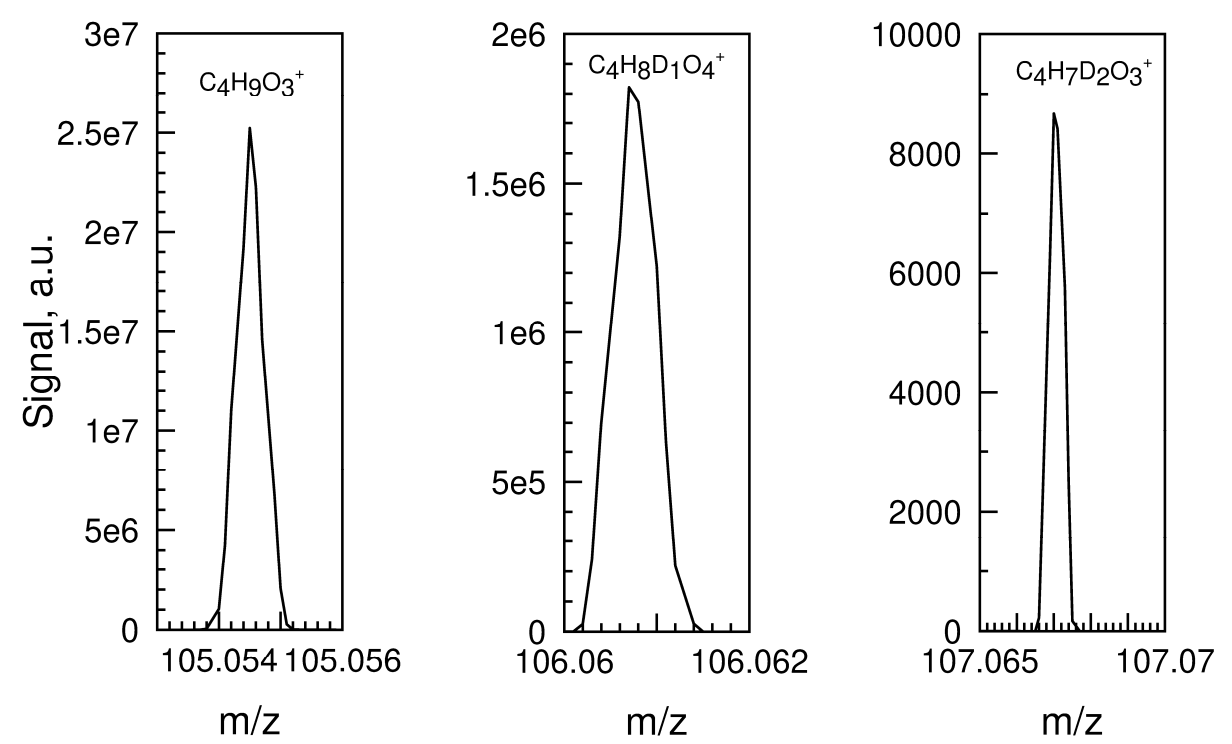

Figure 3. Mass spectrum showing the formation of $\mathrm{C}_{4} \mathrm{H}_{8} \mathrm{D}_{1} \mathrm{O}_{4}{ }^{+}$resulting from $\mathrm{H} / \mathrm{D}$ exchange on $\mathrm{ROOH}$ and diols, and of $\mathrm{C}_{4} \mathrm{H}_{7} \mathrm{D}_{2} \mathrm{O}_{3}{ }^{+}$which indicates the likely presence of diols. One should note that these $\mathrm{D}_{1}$ and $\mathrm{D}_{2}$ ions could not be observed without addition of $\mathrm{D}_{2} \mathrm{O}$ to the sample. Analyses were performed in FIA APCI $(+)$ using the concentrated ( $70 \%$ evaporated) sample obtained by oxidation of THF at $600 \mathrm{~K}$.

Organic dihydroperoxides can be produced via $\mathrm{H}$-atom abstraction by peroxy hydroperoxy radicals:

$$
\mathrm{OOQOOH}+\mathrm{R}^{\prime} \mathrm{H} \rightarrow \mathrm{HOOQOOH}+\mathrm{R}^{\prime}
$$

The signal recorded at $\mathrm{m} / \mathrm{z} 137.0444\left(\mathrm{C}_{4} \mathrm{H}_{9} \mathrm{O}_{5}{ }^{+}\right)$is likely due to the presence of dihydroperoxides in THF oxidation products (Figure 3). 


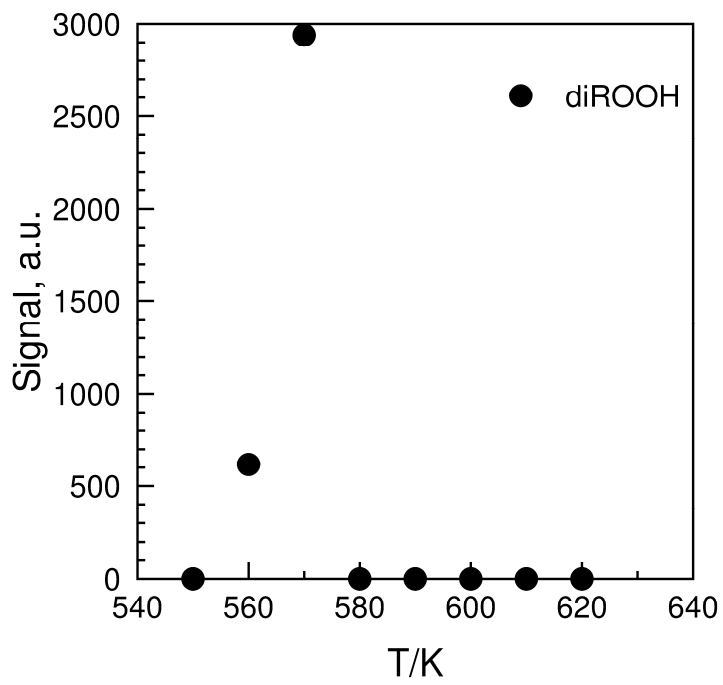

Figure 4. Formation of $\mathrm{C}_{4} \mathrm{H}_{8} \mathrm{O}_{5}$ in a JSR by oxidation of $5000 \mathrm{ppm}$ of THF. The analyses were performed in FIA and $\mathrm{APCI}(+)$ mode. The data (dots) represent the signal recorded at $\mathrm{m} / \mathrm{z} 137.0444\left(\mathrm{C}_{4} \mathrm{H}_{9} \mathrm{O}_{5}{ }^{+}\right)$.

Table 3. Products of THF ( $5000 \mathrm{ppm}$ ) oxidation in a JSR at $600 \mathrm{~K}$ and $10 \mathrm{~atm}$. H/D exchange was observed after addition of $60 \mu \mathrm{L}$ of $\mathrm{D}_{2} \mathrm{O}$ to $200 \mu \mathrm{L}$ of a $70 \%$ evaporated sample (reaction time: $25 \mathrm{~min}$ ). The analyses were performed in FIA/APCI (+/-) modes.

\begin{tabular}{|c|c|c|c|c|c|c|c|}
\hline \multirow{2}{*}{$\begin{array}{l}\text { M } \\
(\mathrm{g} / \mathrm{mol})\end{array}$} & \multicolumn{3}{|c|}{ Species } & \multicolumn{2}{|c|}{ APCI $(+)$} & \multicolumn{2}{|c|}{ APCI $(-)$} \\
\hline & Formula & Name & Ref. & $\begin{array}{l}\mathrm{m} / \mathrm{z} \\
(\mathrm{MH})^{+}\end{array}$ & $\begin{array}{l}\text { Signal } \\
(\mathrm{a} . \mathrm{u})\end{array}$ & $\begin{array}{l}\mathrm{m} / \mathrm{z} \\
(\mathrm{M}-\mathrm{H})^{-}\end{array}$ & $\begin{array}{l}\text { Signal } \\
\text { (a.u) }\end{array}$ \\
\hline 26 & $\mathrm{C}_{2} \mathrm{H}_{2}$ & Acetylene & $\mathrm{c}, \mathrm{e}$ & - & - & $\begin{array}{l}\mathrm{HCOO}^{-} \\
\text {Adduct } \\
71.039\end{array}$ & $2.2 \mathrm{E} 8$ \\
\hline 28 & $\mathrm{C}_{2} \mathrm{H}_{4}$ & Ethene & $\mathrm{b}, \mathrm{c}, \mathrm{e}, \mathrm{f}$ & - & - & $\begin{array}{l}\mathrm{HCOO}^{-} \\
\text {Adduct } \\
73.0296\end{array}$ & $8.2 \mathrm{E} 7$ \\
\hline 30 & $\mathrm{C}_{2} \mathrm{H}_{6}$ & Ethane & $\mathrm{c}, \mathrm{e}$ & - & - & $\begin{array}{l}\mathrm{HCOO}^{-} \\
\text {adduct } \\
75.0452\end{array}$ & $2.7 \mathrm{E} 5$ \\
\hline 30 & $\mathrm{CH}_{2} \mathrm{O}$ & Formaldehyde & $\mathrm{a}, \mathrm{b}, \mathrm{c}, \mathrm{e}$ & - & - & $\begin{array}{l}\mathrm{HCOO}^{-} \\
\text {adduct } \\
75.0088\end{array}$ & $1.2 \mathrm{E} 9$ \\
\hline 32 & $\mathrm{CH}_{4} \mathrm{O}$ & Methanol & $\mathrm{c}$ & - & - & $\begin{array}{l}\mathrm{HCOO}^{-} \\
\text {adduct } \\
77.0245\end{array}$ & $7.0 \mathrm{E} 3$ \\
\hline 40 & $\mathrm{C}_{3} \mathrm{H}_{4} *$ & Propyne or allene & & - & - & $\begin{array}{l}\mathrm{HCOO}^{-} \\
\text {adduct } \\
85.0296\end{array}$ & $5.6 \mathrm{E} 6$ \\
\hline 42 & $\mathrm{C}_{3} \mathrm{H}_{6}$ & $\begin{array}{l}\text { Propene (propylene) } \\
\text { Cyclopropane }\end{array}$ & $\begin{array}{l}a, b, e, f \\
c\end{array}$ & - & - & $\begin{array}{l}\mathrm{HCOO}^{-} \\
\text {adduct } \\
87.0453\end{array}$ & $1.8 \mathrm{E} 6$ \\
\hline 42 & $\mathrm{C}_{2} \mathrm{H}_{2} \mathrm{O}^{*}$ & Ethenone (Ketene) & & - & - & $\begin{array}{l}\mathrm{HCOO}^{-} \\
\text {adduct } \\
87.0089\end{array}$ & $1.8 \mathrm{E} 8$ \\
\hline 44 & $\mathrm{C}_{3} \mathrm{H}_{8}$ & Propane & $\mathrm{a}, \mathrm{e}$ & - & - & $\begin{array}{l}\mathrm{HCOO}^{-} \\
\text {adduct } \\
89.0609\end{array}$ & $3.9 \mathrm{E} 3$ \\
\hline 44 & $\mathrm{C}_{2} \mathrm{H}_{4} \mathrm{O}$ & $\begin{array}{l}\text { Oxirane (Ethylene oxide) } \\
\text { Acetaldehyde }\end{array}$ & $\begin{array}{l}\mathrm{a}, \mathrm{e} \\
\mathrm{c}, \mathrm{e}\end{array}$ & - & - & $\begin{array}{l}\mathrm{HCOO}^{-} \\
\text {adduct }\end{array}$ & $2.9 \mathrm{E} 8$ \\
\hline
\end{tabular}




\begin{tabular}{|c|c|c|c|c|c|c|c|}
\hline & & & & & & 89.0245 & \\
\hline 46 & $\mathrm{CH}_{2} \mathrm{O}_{2}$ & Formic acid & $\mathrm{c}$ & - & - & $\begin{array}{l}\mathrm{HCOO}^{-} \\
\text {adduct } \\
91.0038\end{array}$ & $4.7 \mathrm{E} 7$ \\
\hline 46 & $\mathrm{C}_{2} \mathrm{H}_{6} \mathrm{O}$ & Ethanol & $\mathrm{c}$ & - & - & $\begin{array}{l}\mathrm{HCOO}^{-} \\
\text {adduct } \\
91.0401\end{array}$ & $5.9 \mathrm{E} 3$ \\
\hline 54 & $\mathrm{C}_{3} \mathrm{H}_{2} \mathrm{O}^{*}$ & & & 55.0179 & $7.9 \mathrm{E} 5$ & 53.0033 & $1.3 \mathrm{E} 3$ \\
\hline 54 & $\mathrm{C}_{4} \mathrm{H}_{6}$ & 1,3-Butadiene & $\mathrm{a}, \mathrm{e}$ & 55.0544 & $1.8 \mathrm{E} 7$ & - & - \\
\hline 56 & $\mathrm{C}_{3} \mathrm{H}_{4} \mathrm{O}$ & 2-Propenal (Acrolein) & $a, b, c, e, g$ & 57.0336 & $3.4 \mathrm{E} 7$ & 55.0189 & $5.7 \mathrm{E} 6$ \\
\hline 56 & $\mathrm{C}_{4} \mathrm{H}_{8}$ & 1-Butene & $\mathrm{a}, \mathrm{e}$ & 57.0700 & $1.6 \mathrm{E} 6$ & - & - \\
\hline 58 & $\mathrm{C}_{2} \mathrm{H}_{2} \mathrm{O}_{2} *$ & & & 59.0128 & $5.0 \mathrm{E} 4$ & 56.9983 & $1.9 \mathrm{E} 3$ \\
\hline 58 & $\mathrm{C}_{3} \mathrm{H}_{6} \mathrm{O}$ & $\begin{array}{l}\text { 2-Methyloxirane } \\
\text { Acetone } \\
\text { Propanal } \\
\text { Oxetane } \\
\text { Methylvinylether } \\
\text { Propenol }\end{array}$ & $\begin{array}{l}\text { a,c,i } \\
\mathrm{a} \\
\mathrm{a}, \mathrm{e}, \mathrm{i} \\
\mathrm{a} \\
\mathrm{b} \\
\mathrm{i} \\
\end{array}$ & 59.0492 & $5.3 \mathrm{E} 6$ & 57.0346 & $6.0 \mathrm{E} 5$ \\
\hline 59 & $\mathrm{C}_{3} \mathrm{H}_{5} \mathrm{D}_{1} \mathrm{O}^{*}$ & Propenol-d1 & & 60.0555 & $1.1 \mathrm{E} 4$ & 58.0409 & $3.3 \mathrm{E} 3$ \\
\hline 60 & $\mathrm{C}_{2} \mathrm{H}_{4} \mathrm{O}_{2}$ & $\begin{array}{l}\text { Acetic acid } \\
\text { Methyl formate }\end{array}$ & $\begin{array}{l}\mathrm{c} \\
\mathrm{c}\end{array}$ & 61.0284 & $8.4 \mathrm{E} 6$ & 59.0139 & $1.9 \mathrm{E} 7$ \\
\hline 61 & $\mathrm{C}_{2} \mathrm{H}_{3} \mathrm{D}_{1} \mathrm{O}_{2} *$ & Acetic acid- $\mathrm{d}_{1}$ & & 62.0347 & $5.1 \mathrm{e} 5$ & 60.0202 & $1.3 \mathrm{E} 6$ \\
\hline 62 & $\mathrm{C}_{2} \mathrm{H}_{6} \mathrm{O}_{2}{ }^{*}$ & & & 63.0441 & $4.3 \mathrm{E} 3$ & 61.0296 & $3.0 \mathrm{E} 4$ \\
\hline 68 & $\mathrm{C}_{4} \mathrm{H}_{4} \mathrm{O}$ & Furan & $\mathrm{a}, \mathrm{b}, \mathrm{c}, \mathrm{e}$ & 69.0334 & $1.2 \mathrm{E} 7$ & 67.0190 & $1.2 \mathrm{E} 4$ \\
\hline 70 & $\mathrm{C}_{4} \mathrm{H}_{6} \mathrm{O}$ & $\begin{array}{l}\text { 2,3-DHF } \\
\text { 2,5-DHF } \\
\text { Cyclo-propane- } \\
\text { carboxaldehyde } \\
\text { 2-Butenal }\end{array}$ & $\begin{array}{l}a, b, e, f, i \\
a, b, f, i \\
a, e \\
a, e\end{array}$ & 71.0491 & $1.6 \mathrm{E} 9$ & 69.0347 & $1.1 \mathrm{E} 6$ \\
\hline 70 & $\mathrm{C}_{3} \mathrm{H}_{2} \mathrm{O}_{2} *$ & & & 71.0127 & $7.8 \mathrm{E} 5$ & 68.9983 & $8.5 \mathrm{E} 5$ \\
\hline 72 & $\mathrm{C}_{4} \mathrm{H}_{8} \mathrm{O}$ & THF & & 73.0647 & $2.5 \mathrm{E} 8$ & 71.0503 & $3.5 \mathrm{E} 5$ \\
\hline 72 & $\mathrm{C}_{3} \mathrm{H}_{4} \mathrm{O}_{2} *$ & & & 73.0283 & $7.8 \mathrm{E} 7$ & 71.0139 & $2.2 \mathrm{E} 8$ \\
\hline 74 & $\mathrm{C}_{3} \mathrm{H}_{6} \mathrm{O}_{2}$ & Ethyl formate & $\mathrm{c}$ & 75.0440 & $1.2 \mathrm{E} 7$ & 73.0296 & $8.2 \mathrm{E} 7$ \\
\hline 82 & $\mathrm{C}_{4} \mathrm{H}_{2} \mathrm{O}_{2} *$ & & & 83.0126 & $8.3 \mathrm{E} 5$ & - & - \\
\hline 76 & $\mathrm{C}_{3} \mathrm{H}_{8} \mathrm{O}_{2} *$ & & & 77.0596 & $3.0 \mathrm{E} 6$ & 75.0452 & $2.8 \mathrm{E} 5$ \\
\hline 76 & $\mathrm{C}_{2} \mathrm{H}_{4} \mathrm{O}_{3} *$ & & & 77.0232 & $2.1 \mathrm{E} 4$ & 75.0088 & $1.1 \mathrm{E} 9$ \\
\hline 83 & $\mathrm{C}_{4} \mathrm{H}_{1} \mathrm{D}_{1} \mathrm{O}_{2}$ * & & & - & $\S$ & - & $\S$ \\
\hline 84 & $\mathrm{C}_{4} \mathrm{H}_{4} \mathrm{O}_{2}$ & 1,4-Dioxine & $\mathrm{a}, \mathrm{e}$ & 85.0283 & $5.0 \mathrm{E} 8$ & 83.0141 & $1.5 \mathrm{E} 6$ \\
\hline 85 & $\mathrm{C}_{4} \mathrm{H}_{5} \mathrm{O}_{2} *$ & & $\mathrm{~d}$ & 86.0361 & $9.7 \mathrm{E} 4$ & 84.0218 & $3.0 \mathrm{E} 3$ \\
\hline 86 & $\mathrm{C}_{4} \mathrm{H}_{6} \mathrm{O}_{2}$ & $\begin{array}{l}\text { Formic acid, 2-propenyl ester } \\
\text { Butanedial } \\
\gamma \text {-Butyrolactone } \\
\text { THF-3-one } \\
\text { 3,4-Epoxy-THF } \\
\text { 1,3 Dioxene } \\
\text { 1,4 Dioxene }\end{array}$ & $\begin{array}{l}\text { a } \\
\text { a,f,e,g,i } \\
\text { a,b,c,i } \\
\text { b } \\
\text { f,i } \\
\text { e } \\
\text { a } \\
\end{array}$ & 87.0440 & $1.2 \mathrm{E} 9$ & 85.0296 & $5.6 \mathrm{E} 6$ \\
\hline 88 & $\mathrm{C}_{4} \mathrm{H}_{8} \mathrm{O}_{2}$ & 1,4-Dioxane & & 89.0596 & $1.2 \mathrm{E} 7$ & 87.0452 & $2.1 \mathrm{E} 6$ \\
\hline 90 & $\mathrm{C}_{3} \mathrm{H}_{6} \mathrm{O}_{3} *$ & & & 91.0389 & $9.6 \mathrm{E} 6$ & 89.0245 & $2.9 \mathrm{E} 8$ \\
\hline 90 & $\mathrm{C}_{4} \mathrm{H}_{10} \mathrm{O}_{2} *$ & & & 91.0752 & $8.0 \mathrm{E} 5$ & 89.0610 & $3.9 \mathrm{E} 3$ \\
\hline 91 & $\mathrm{C}_{4} \mathrm{H}_{9} \mathrm{D}_{1} \mathrm{O}_{2}$ & & & - & $\S$ & - & $\S$ \\
\hline 92 & $\mathrm{C}_{7} \mathrm{H}_{8} *$ & Toluene and isomers & & 93.0698 & $3.2 \mathrm{E} 6$ & - & - \\
\hline 98 & $\mathrm{C}_{4} \mathrm{H}_{2} \mathrm{O}_{3} *$ & & & 99.0075 & $9.6 \mathrm{E} 4$ & 96.9932 & $1.0 \mathrm{E} 5$ \\
\hline 99 & $\mathrm{C}_{4} \mathrm{H}_{1} \mathrm{D}_{1} \mathrm{O}_{3} *$ & & & - & $\S$ & - & $\S$ \\
\hline 100 & $\mathrm{C}_{4} \mathrm{H}_{4} \mathrm{O}_{3}{ }^{*}$ & Diketones and isomers & & 101.0232 & $5.3 \mathrm{E} 6$ & 99.0089 & $6.2 \mathrm{E} 8$ \\
\hline
\end{tabular}




\begin{tabular}{|c|c|c|c|c|c|c|c|}
\hline 101 & $\mathrm{C}_{4} \mathrm{H}_{3} \mathrm{D}_{1} \mathrm{O}_{3} *$ & Diketones isomers & & 102.0295 & $5.5 \mathrm{E} 4$ & 100.0152 & $4.3 \mathrm{E} 6$ \\
\hline 102 & $\mathrm{C}_{4} \mathrm{H}_{6} \mathrm{O}_{3}$ & 2,3 DHF-OOH & $b, e, f, i$ & 103.0388 & 9.3E7 & 101.0245 & $1.0 \mathrm{E} 8$ \\
\hline 103 & $\mathrm{C}_{4} \mathrm{H}_{5} \mathrm{D}_{1} \mathrm{O}_{3}$ * & 2,3 DHF-OOH-d ${ }_{1}$ & & 104.0451 & $5.7 \mathrm{E} 6$ & 102.0308 & $1.9 \mathrm{E} 6$ \\
\hline 104 & $\mathrm{C}_{4} \mathrm{H}_{8} \mathrm{O}_{3}$ & Diols and/or ROOH & $\mathrm{c}, \mathrm{e}$ & 105.0545 & $2.5 \mathrm{E} 7$ & 103.0402 & $1.7 \mathrm{E} 7$ \\
\hline 105 & $\mathrm{C}_{4} \mathrm{H}_{7} \mathrm{D}_{1} \mathrm{O}_{3}$ & Diols-d $_{1}$ and/or ROOH-d $\mathrm{d}_{1}$ & & 106.0608 & $1.8 \mathrm{E} 6$ & 104.0465 & $1.1 \mathrm{E} 6$ \\
\hline 106 & $\mathrm{C}_{4} \mathrm{H}_{6} \mathrm{D}_{2} \mathrm{O}_{3}$ & Diols- $\mathrm{d}_{2}$ & & 107.0671 & $8.9 \mathrm{E} 3$ & 105.0526 & $\S$ \\
\hline 106 & $\mathrm{C}_{4} \mathrm{H}_{10} \mathrm{O}_{3} *$ & & & 107.0701 & $1.1 \mathrm{E} 5$ & 105.0559 & $3.4 \mathrm{E} 3$ \\
\hline 107 & $\mathrm{C}_{4} \mathrm{H}_{9} \mathrm{D}_{1} \mathrm{O}_{3}$ * & & & - & - & - & - \\
\hline 116 & $\mathrm{C}_{4} \mathrm{H}_{4} \mathrm{O}_{4} *$ & & & 117.0181 & $1.8 \mathrm{E} 6$ & 115.0039 & $3.8 \mathrm{E} 8$ \\
\hline 117 & $\mathrm{C}_{4} \mathrm{H}_{3} \mathrm{D}_{1} \mathrm{O}_{4} *$ & & & 118.0244 & $1.8 \mathrm{E} 5$ & 116.0101 & $2.5 \mathrm{E} 7$ \\
\hline 118 & $\mathrm{C}_{4} \mathrm{H}_{2} \mathrm{D}_{2} \mathrm{O}_{4}$ & & & - & - & - & - \\
\hline 118 & $\mathrm{C}_{4} \mathrm{H}_{6} \mathrm{O}_{4}$ & $\begin{array}{l}\text { KHP } \\
\text { Succinic acid }\end{array}$ & $\begin{array}{l}\mathrm{b}, \mathrm{d}, \mathrm{f} \\
\mathrm{c}\end{array}$ & $\begin{array}{l}119.0338 \\
-\end{array}$ & $\begin{array}{l}3.5 \mathrm{E} 6 \\
-\end{array}$ & $\begin{array}{l}117.0195 \\
-\end{array}$ & $\begin{array}{l}1.2 \mathrm{E} 9 \\
-\quad \\
\end{array}$ \\
\hline 119 & $\mathrm{C}_{4} \mathrm{H}_{5} \mathrm{D}_{1} \mathrm{O}_{4} *$ & KHP-d $_{1}$, Succinic acid- $_{1}$ & & 120.0401 & $8.9 \mathrm{E} 4$ & 118.0257 & $7.6 \mathrm{E} 7$ \\
\hline 120 & $\mathrm{C}_{4} \mathrm{H}_{4} \mathrm{D}_{2} \mathrm{O}_{4} *$ & Succinic acid- $\mathrm{d}_{2}$ & & - & $\S$ & 119.0319 & $2.6 \mathrm{E} 4$ \\
\hline 120 & $\mathrm{C}_{4} \mathrm{H}_{8} \mathrm{O}_{4} *$ & & & 121.0495 & $1.4 \mathrm{E} 6$ & 119.0350 & $2.3 \mathrm{E} 7$ \\
\hline 121 & $\mathrm{C}_{4} \mathrm{H}_{7} \mathrm{D}_{1} \mathrm{O}_{4} *$ & & & - & - & 120.0414 & $3.6 \mathrm{E} 6$ \\
\hline 122 & $\mathrm{C}_{4} \mathrm{H}_{6} \mathrm{D}_{2} \mathrm{O}_{4} *$ & & & - & - & 121.0477 & $8.2 \mathrm{E} 4$ \\
\hline 132 & $\mathrm{C}_{4} \mathrm{H}_{4} \mathrm{O}_{5} *$ & Di keto hydroperoxide & & - & - & 130.9987 & $6.1 \mathrm{E} 6$ \\
\hline 133 & $\mathrm{C}_{4} \mathrm{H}_{3} \mathrm{D}_{1} \mathrm{O}_{5}$ * & Di keto hydroperoxide- $\mathrm{d}_{1}$ & & - & - & 132.0050 & $3.0 \mathrm{E} 4$ \\
\hline 134 & $\mathrm{C}_{4} \mathrm{H}_{6} \mathrm{O}_{5} *$ & & & 135.0288 & $4.8 \mathrm{E} 3$ & 133.0144 & $5.7 \mathrm{E} 7$ \\
\hline 135 & $\mathrm{C}_{4} \mathrm{H}_{5} \mathrm{D}_{1} \mathrm{O}_{5}$ * & & & - & - & 134.0207 & $7.1 \mathrm{E} 6$ \\
\hline 136 & $\mathrm{C}_{4} \mathrm{H}_{4} \mathrm{D}_{2} \mathrm{O}_{5}$ * & & & - & - & 135.0270 & $1.3 \mathrm{E} 5$ \\
\hline 137 & $\mathrm{C}_{4} \mathrm{H}_{3} \mathrm{D}_{3} \mathrm{O}_{5} *$ & & & - & - & 136.0334 & $1.2 \mathrm{E} 4$ \\
\hline 136 & $\mathrm{C}_{4} \mathrm{H}_{8} \mathrm{O}_{5} *$ & di $\mathrm{ROOH}$ & & - & - & 135.0301 & $2.7 \mathrm{E} 6$ \\
\hline 137 & $\mathrm{C}_{4} \mathrm{H}_{7} \mathrm{D}_{1} \mathrm{O}_{5} *$ & di ROOH-d ${ }_{1}$ & & - & - & 136.0363 & $2.1 \mathrm{E} 5$ \\
\hline 138 & $\mathrm{C}_{4} \mathrm{H}_{6} \mathrm{D}_{2} \mathrm{O}_{5}$ * & di-ROOH-d 2 & & - & - & 137.0427 & $1.8 \mathrm{E} 3$ \\
\hline 150 & $\mathrm{C}_{4} \mathrm{H}_{6} \mathrm{O}_{6} *$ & Keto di-hydroperoxide & & - & - & 149.0093 & $1.9 \mathrm{E} 6$ \\
\hline 151 & $\mathrm{C}_{4} \mathrm{H}_{5} \mathrm{D}_{1} \mathrm{O}_{6} *$ & Keto di-hydroperoxide- $\mathrm{d}_{1}$ & & - & - & 150.0156 & $3.8 \mathrm{E} 4$ \\
\hline 152 & $\mathrm{C}_{4} \mathrm{H}_{4} \mathrm{D}_{2} \mathrm{O}_{6} *$ & Keto di-hydroperoxide- $\mathrm{d}_{2}$ & & - & - & - & $\S$ \\
\hline 168 & $\mathrm{C}_{4} \mathrm{H}_{8} \mathrm{O}_{7} *$ & tri $\mathrm{ROOH}$ & & - & - & 167.0191 & $2.0 \mathrm{E} 6$ \\
\hline 169 & $\mathrm{C}_{4} \mathrm{H}_{7} \mathrm{D}_{1} \mathrm{O}_{7} *$ & tri $\mathrm{ROOH}-\mathrm{d}_{1}$ & & - & - & 168.0264 & $5.4 \mathrm{E} 5$ \\
\hline 170 & $\mathrm{C}_{4} \mathrm{H}_{6} \mathrm{D}_{2} \mathrm{O}_{7} *$ & tri $\mathrm{ROOH}-\mathrm{d}_{2}$ & & - & - & - & $\S$ \\
\hline 171 & $\mathrm{C}_{4} \mathrm{H}_{5} \mathrm{D}_{3} \mathrm{O}_{7} *$ & tri $\mathrm{ROOH}-\mathrm{d}_{3}$ & & - & - & - & $\S$ \\
\hline
\end{tabular}

Note: $\gamma$-Butyrolactone, THF-3-one, 2,3-DHF, 2,5-DHF, succinic acid, cyclopropane carboxaldehyde, 3,4-epoxy tetrahydrofuran and furan identification was checked by UHPLC-MS-MS analysis of pure chemicals in acetonitrile (succinic acid was not found); * newly detected species (see Supporting information for structures, Table S3); § Below detection limit. References: $\mathrm{a}^{18} ; \mathrm{b}^{58} ; \mathrm{c}^{17} \mathrm{~d}^{24} ; \mathrm{e}^{22} ; \mathrm{f}^{59} ; \mathrm{g}^{21} ; \mathrm{h}^{60}, \mathrm{i}^{6}{ }^{61}$

Ketohydroperoxides $\left(\mathrm{C}_{4} \mathrm{H}_{6} \mathrm{O}_{4}\right)$ are important products formed in cool flames. $\mathrm{H} / \mathrm{D}$ exchange using $\mathrm{D}_{2} \mathrm{O}$ was also used to verify the presence of an $-\mathrm{OOH}$ group in $\mathrm{C}_{4} \mathrm{H}_{6} \mathrm{O}_{4}$ products (Table 3). Figure 5 shows a typical example of hydrogen-deuterium exchange observed for $\mathrm{C}_{4} \mathrm{H}_{6} \mathrm{O}_{4}$. One can see the formation of the $\mathrm{C}_{4} \mathrm{H}_{4} \mathrm{DO}_{4}{ }^{-}$ion. Succinic acid, $\left(\mathrm{CH}_{2}\right)_{2}(\mathrm{C}(=\mathrm{O}) \mathrm{OH})_{2}$, has been reported earlier as product of THF oxidation. It could be responsible for the very minor second $\mathrm{H} / \mathrm{D}$ exchange $\left(\mathrm{C}_{4} \mathrm{H}_{3} \mathrm{D}_{2} \mathrm{O}_{4}{ }^{-}\right)$observed here (Table 3). However, HPLC analyses of all samples did not show a chromatographic peak at $10.4 \mathrm{~min}$, the retention time at which pure succinic acid in solution in ACN was observed. 

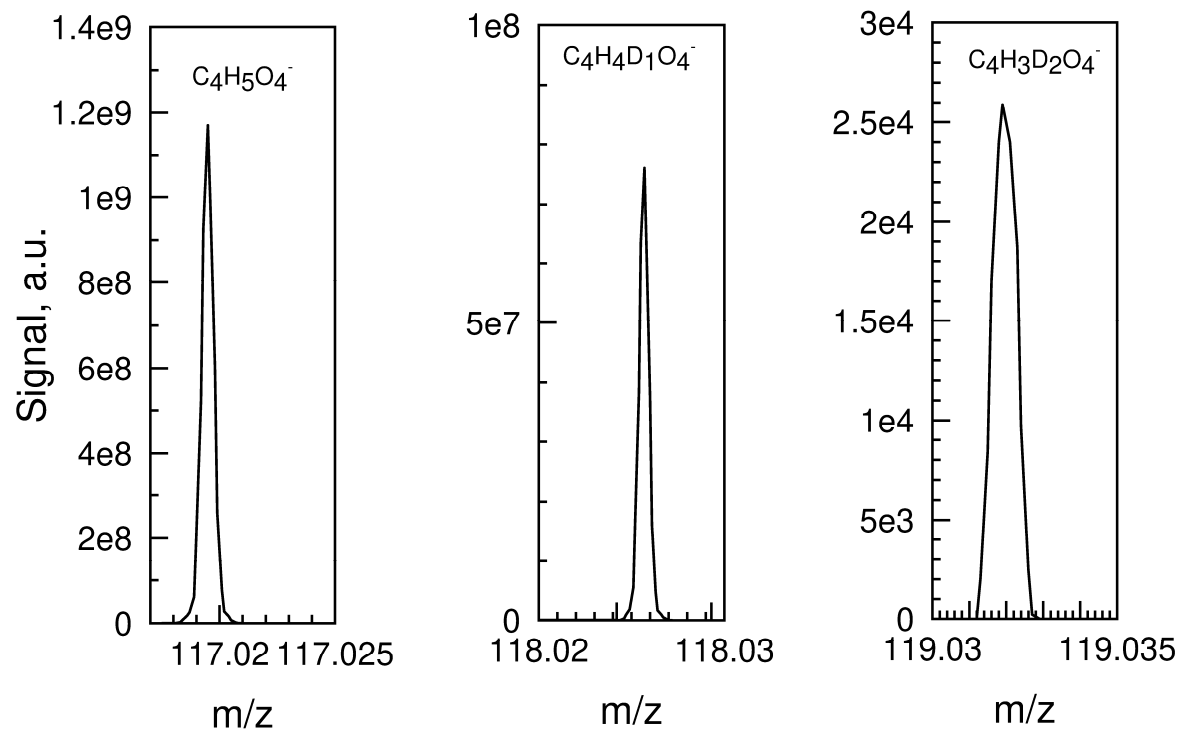

Figure 5. Mass spectrum showing the formation of $\mathrm{C}_{4} \mathrm{H}_{4} \mathrm{D}_{1} \mathrm{O}_{4}{ }^{-}$resulting from $\mathrm{H} / \mathrm{D}$ exchange on KHPs and of $\mathrm{C}_{4} \mathrm{H}_{3} \mathrm{D}_{2} \mathrm{O}_{4}{ }^{-}$which indicates the possible minor presence of succinic acid. One should note that these deuterated ions could not be observed without addition of $\mathrm{D}_{2} \mathrm{O}$ to the sample. The analyses were performed in FIA APCI (-) using the concentrated (70\% evaporated) sample obtained by oxidation of THF at $600 \mathrm{~K}$.

The total formation of KHPs was used to test the kinetic model. Figure 6a shows a fair representation of the qualitative data was obtained. This is also true for THF (Figure 5b).
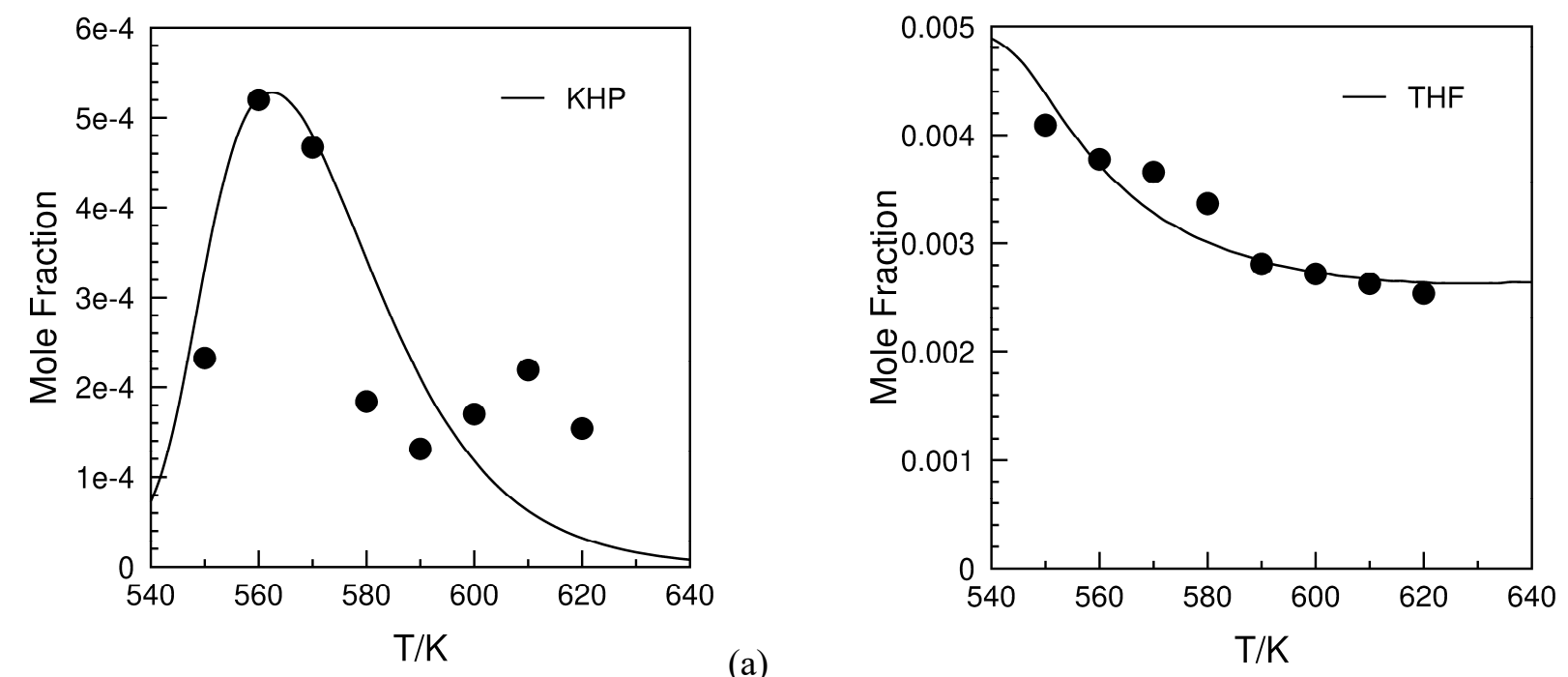

(a)

(b)

Figure 6. (a) Formation of $\mathrm{C}_{4} \mathrm{H}_{6} \mathrm{O}_{4}$ in a JSR by oxidation of $5000 \mathrm{ppm}$ of THF. Analyses were performed in FIA and APCI $(+)$ mode. The data (dots) represent the signal recorded at $\mathrm{m} / \mathrm{z} 119.0338\left(\mathrm{C}_{4} \mathrm{H}_{7} \mathrm{O}_{4}{ }^{+}\right)$, scaled to the KHPs maximum computed mole fraction. (b) Consumption of THF under the same conditions based on $\mathrm{m} / \mathrm{z} 73.0647$ $\left(\mathrm{C}_{4} \mathrm{H}_{9} \mathrm{O}^{+}\right)$. The data (dots) are compared to simulations (lines).

Figure 7 shows that UHPLC-MS analyses with APCI $(+)$ allowed separating most of the ketohydroperoxides isomers. The examination of the chromatogram seems to indicate the presence of 5 to 6 isomers ( $\mathrm{Rt}=4.28$, $\mathrm{Rt}=4.49, \mathrm{Rt}=4.53$, likely coelution at $\mathrm{Rt}=5.06$, and $\mathrm{Rt}=5.20$ ). According to the model of Fénard et al., ${ }^{22}$ the most 
abundant KHP by far is the $\alpha \alpha^{\prime}$ isomer, followed by the $\alpha \beta^{\prime}$ and $\beta \alpha^{\prime}$ isomers, the three others $\left(\alpha \beta>\beta \alpha>\beta \beta^{\prime}\right)$ being negligeable (mole fractions of $10^{-9}-10^{-12}$ ).

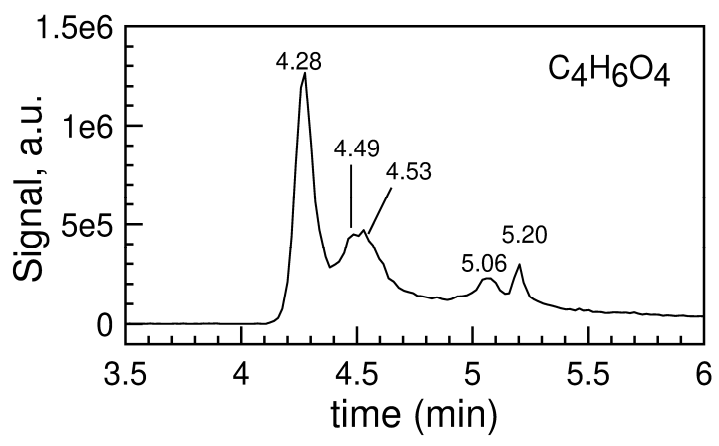

Figure 7. Chromatographic separation on a Hypercarb PGC column $\left(100 \% \mathrm{ACN}, 100 \mu \mathrm{L} / \mathrm{min}, 40^{\circ} \mathrm{C}\right)$ of $\mathrm{KHPs}$ isomers $\left(\mathrm{C}_{4} \mathrm{H}_{6} \mathrm{O}_{4}\right)$ obtained by THF oxidation at $590 \mathrm{~K}$. The APCI + mode was used. Isomers structures are given in Table 2.

MS/MS analyses were carried out to identify the ketohydroperoxides produced during THF low-temperature oxidation (Supporting information, Table S4). Fragmentation at 10,30, 50, and $70 \mathrm{eV}$ was used, but did not allow discriminating the isomers due to very similar fragmentation patterns. (Supporting information Table S5).

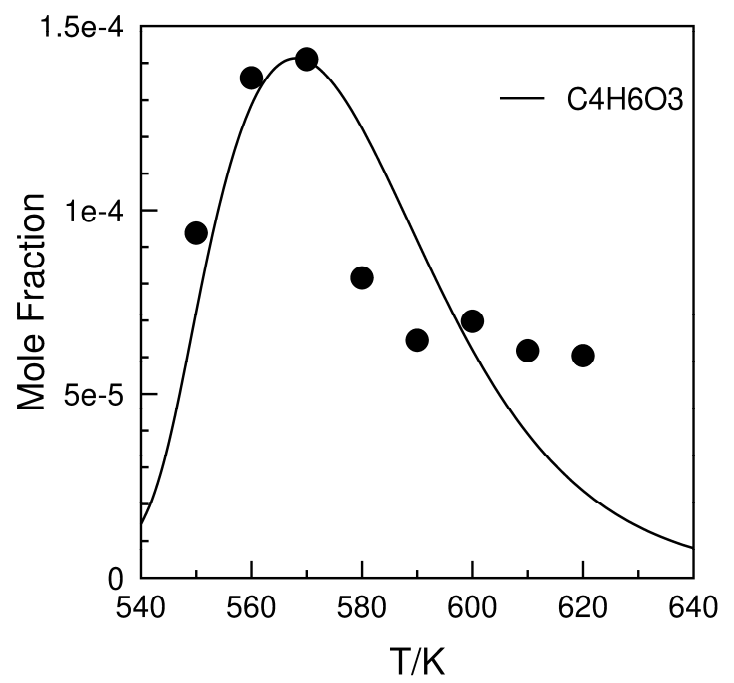

Figure 8. Formation of $\mathrm{C}_{4} \mathrm{H}_{6} \mathrm{O}_{3}$ in a JSR during the oxidation of $5000 \mathrm{ppm}$ of THF. Analyses were performed in FIA and APCI $(+)$ mode. The data (dots) represent the signal recorded at $\mathrm{m} / \mathrm{z} 103.0388\left(\mathrm{C}_{4} \mathrm{H}_{7} \mathrm{O}_{3}{ }^{+}\right)$, scaled to the maximum computed mole fraction (line).

Dihydrofuran hydroperoxides $\left(\mathrm{C}_{4} \mathrm{H}_{6} \mathrm{O}_{3}\right)$, which are formed by decomposition of OOQOOH, according to the modeling, were observed. The present qualitative data were compared to simulations showing fair agreement in terms of variation of MS signal intensity with oxidation temperature (Figure 8).

Dihydrofurans can be formed by decomposition of $\mathrm{RO}_{2}$ and $\mathrm{QOOH}$ in cool flames ${ }^{22}$ :

$$
\begin{gathered}
\mathrm{RO}_{2} \rightarrow \text { dihydrofuran }+\mathrm{HO}_{2} \\
\mathrm{QOOH} \rightarrow \text { dihydrofuran }+\mathrm{HO}_{2}
\end{gathered}
$$


They were observed in the present study. Our qualitative data were compared to simulations showing fair agreement in terms of variation of MS signal intensity with oxidation temperature (Figure 9).

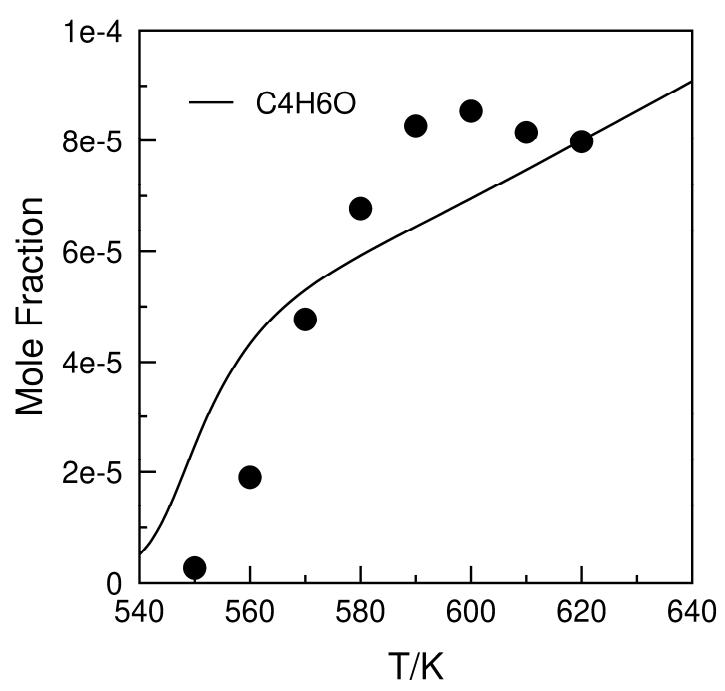

Figure 9. Formation of $\mathrm{C}_{4} \mathrm{H}_{6} \mathrm{O}$ in a JSR by oxidation of $5000 \mathrm{ppm}$ of THF. Analyses were performed in UHPLC and APCI $(+)$ mode. The data (dots) represent the signal recorded at $\mathrm{m} / \mathrm{z} 71.0491\left(\mathrm{C}_{4} \mathrm{H}_{7} \mathrm{O}^{+}\right)$, scaled to the maximum computed mole fraction (line).

$\gamma$-Butyrolactone, tetrahydrofuran 3-one, 3, 4-epoxy tetrahydrofuran, succinaldehyde (butanedial), and formic acid 2-propyl ester with formula $\mathrm{C}_{4} \mathrm{H}_{6} \mathrm{O}_{2}$ have been reported as products of THF low-temperature oxidation (Table 3). Injection of pure samples and analyses by UHPLC-MS were used for their characterization. The data showed that $\gamma$-butyrolactone and tetrahydrofuran-3-one, resulting from the oxidation on $\alpha$ and $\beta$ tetrahydrofuranyl radicals, respectively, are the main $\mathrm{C}_{4} \mathrm{H}_{6} \mathrm{O}_{2}$ products.

Figure 10 shows the recorded $\mathrm{m} / \mathrm{z}$ signal vs. THF oxidation temperature. Since the chemical kinetic model ${ }^{22}$ does not distinguish between the different isomers, the total signal at $\mathrm{m} / \mathrm{z} 87.0440$ was used for comparison of data and simulations. One can see from Figure 10 that simulations and data show similar trend.

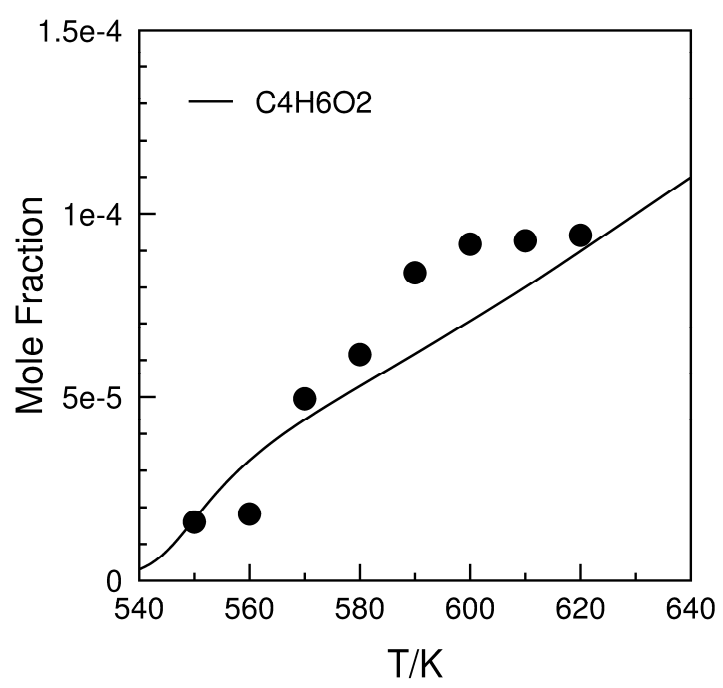


Figure 10. Global formation of $\mathrm{C}_{4} \mathrm{H}_{6} \mathrm{O}_{2}$ in a JSR by oxidation of $5000 \mathrm{ppm}$ of THF. Analyses were performed in FIA and APCI $(+)$ mode. The data (dots) represent the signal recorded at $\mathrm{m} / \mathrm{z} 87.0440\left(\mathrm{C}_{4} \mathrm{H}_{7} \mathrm{O}_{2}{ }^{+}\right)$, scaled to the maximum computed mole fraction (line).

Diketones and isomers $\left(\mathrm{C}_{4} \mathrm{H}_{4} \mathrm{O}_{3}\right)$ were observed here (Table 3). It is generally considered they derive from KHPs. Several routes to diketones have been proposed in the literature, including decomposition of KHPs, ${ }^{62} \mathrm{H}-$ atom abstraction on KHPs, ${ }^{63}$ and roaming reaction of $\mathrm{KHPs}^{64}$. Formation of diketones by decomposition of ${ }^{\circ} \mathrm{OOQOOH}{ }^{65}$ has also been proposed. One can expect the formation of four diketones by oxidation of THF. Figure 11 shows a chromatogram obtained by UHPLC-MS. One can see that several $\mathrm{C}_{4} \mathrm{H}_{4} \mathrm{O}_{3}$ isomers were formed.

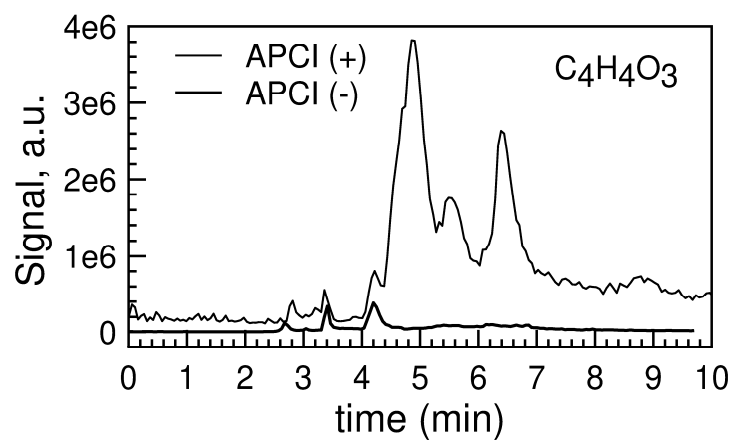

Figure 11. Chromatogram for $\mathrm{C}_{4} \mathrm{H}_{4} \mathrm{O}_{3}$ showing the presence of different isomers. The HPLC-MS data were obtained in APCI + and - (m/z 101.0232 and 99.0089, respectively). A C18 column was used (100\% water, 100 $\left.\mu \mathrm{L} / \mathrm{min}, 40^{\circ} \mathrm{C}\right)$.

Chromatographic peaks obtained in APCI $(+)$ with retention times $>4.5$ min show almost no signal in APCI (-) correspond to compounds which cannot be deprotonated easily. They likely correspond to diketones. Fragmentation of these compounds gave almost undistinguishable fragmentation patterns. The 3 chromatographic peaks observed in APCI (-) at retention time 2.5-4.5 min correspond to compounds for which protonation and deprotonation are possible. We could not identify them presently by MS/MS but the observed H/D exchange (Table 3) could indicate the presence of ketoenols, reported in a recent study of n-pentane oxidation ${ }^{66}$.

Highly oxygenated molecules ( $\mathrm{MW}=132-168)$, deriving from further peroxidation, were also observed (Table 3). H/D exchange was used to confirm the presence of multiple hydroperoxy groups. These products resulted from 3 successive additions of $\mathrm{O}_{2}$ on fuel's radicals. Among these species, one founds diketohydroperoxides ( $\mathrm{MW}=132)$, ketodihydroperoxides ( $\mathrm{MW}=150)$, and tri-hydroperoxides $(\mathrm{MW}=168)$, all reported for the first time as products of THF oxidation. Figure 12 shows an example of the variation of the MS signal as a function of temperature for $\mathrm{C}_{4} \mathrm{H}_{8} \mathrm{O}_{7}$. 


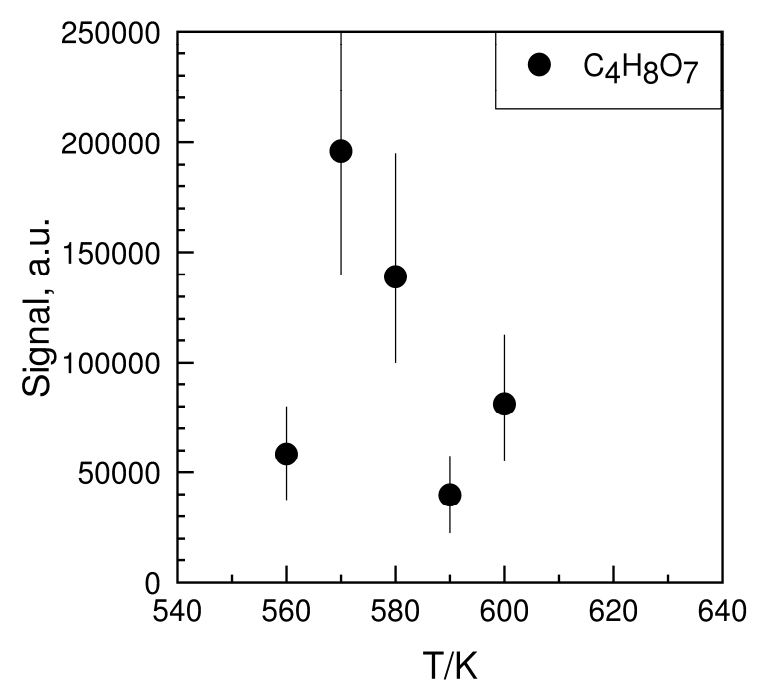

Figure 12. Formation of $\mathrm{C}_{4} \mathrm{H}_{8} \mathrm{O}_{7}$ in a JSR by oxidation of $5000 \mathrm{ppm}$ of THF. Analyses were performed in FIA and APCI (-) mode. The data (symbols) represent the signal recorded at m/z $167.0191\left(\mathrm{C}_{4} \mathrm{H}_{7} \mathrm{O}_{7}{ }^{-}\right)$.

As observed for other low-temperature oxygenated products, except $\mathrm{C}_{4} \mathrm{H}_{6} \mathrm{O}$ and $\mathrm{C}_{4} \mathrm{H}_{6} \mathrm{O}_{2}$, the signal peaks near $570 \mathrm{~K}$.

\section{CONCLUSION AND PERSPECTIVES}

THF oxidation was carried out in a jet-stirred reactor $(10 \mathrm{~atm}$, residence time of $2 \mathrm{~s}, 550-620 \mathrm{~K}$, equivalence ratios of 0.5 , and initial fuel mole fraction of $5000 \mathrm{ppm}$ ). Low-temperature products of THF oxidation were characterized by collecting samples dissolved in acetonitrile for analyses by several analytical techniques (flow injection analyses, UHPLC and HPLC analyses, high resolution mass spectrometry with APCI (+) and (-), and MS-MS). Thanks to these analyses, we could characterize hydroperoxides $\left(\mathrm{C}_{7} \mathrm{H}_{16} \mathrm{O}_{2}\right)$, ketohydroperoxides $\left(\mathrm{C}_{7} \mathrm{H}_{14} \mathrm{O}_{3}\right)$, carboxylic acids, diketones, and highly oxygenated molecules (MW 132, 136, 150, and 168, i.e., $\mathrm{C}_{4} \mathrm{H}_{4} \mathrm{O}_{5}, \mathrm{C}_{4} \mathrm{H}_{8} \mathrm{O}_{5}, \mathrm{C}_{4} \mathrm{H}_{6} \mathrm{O}_{6}$, and $\mathrm{C}_{4} \mathrm{H}_{8} \mathrm{O}_{7}$ ) resulting from the addition up to three $\mathrm{O}_{2}$ molecules on fuel's radicals. To confirm the presence of hydroxy or hydroperoxy groups in the products of THF oxidation, H/D exchange with $\mathrm{D}_{2} \mathrm{O}$ was used.

Twenty-four new products of THF low-temperature oxidation could be detected in this study. These newly detected species are generally not included in the most recent detailed kinetic reaction mechanism for THF oxidation $^{22}$ used here. Their inclusion could be of interest for improving it. As reported by Hansen et al. ${ }^{24}$, it is clear that the relative importance of pathways yielding KHP isomers needs to be reconsidered. This would involve more measurements and high-level computations.

Future studies would benefit from a combination of state-of-the-art techniques such as synchrotron-based mass spectrometry, ultra-high-pressure liquid chromatography and gas chromatography coupled to highresolution mass spectrometry. Furthermore, experiments under more practical conditions, e.g., RCM and/or piston engines, and detailed analyses of exhausts could be performed for further judging the importance of the lowtemperature oxidation products detected in this work. 
The authors gratefully acknowledge funding from the Labex Caprysses (convention ANR-11-LABX-0006-01) and from the Region Centre Val de Loire, EFRD, and CPER (projects PROMESTOCK and APROPOR-E). We thank Prof. P. Favetta, University of Orléans, for lending us a chromatographic column.

\section{ASSOCIATED CONTENT}

Supporting Information

\section{REFERENCES}

(1) Leppard, W. R., The Autoignition Chemistry of n-Butane: An Experimental Study. SAE International: 1987.

(2) Leppard, W. R., The Autoignition Chemistry of Isobutane: A Motored Engine Study. SAE International: 1988.

(3) Leppard, W. R., A Comparison of Olefin and Paraffin Autoignition chemistries: A Motored-Engine Study. SAE International: 1989.

(4) Leppard, W. R., The Autoignition Chemistries of Octane-Enhancing Ethers and Cyclic Ethers: A Motored Engine Study. SAE International: 1991.

(5) Fish, A.; Haskell, W. W.; Read, I. A., The non-isothermal oxidation of 2-methylpentane. - III. The reaction at high pressure. Proceedings of the Royal Society of London. A. Mathematical and Physical Sciences 1969, 313 (1513), 261.

(6) Dagaut, P.; Reuillon, M.; Cathonnet, M., High-Pressure Oxidation of Liquid Fuels from Low to High-Temperature .1. NHeptane and Isooctane. Combust. Sci. Technol. 1994, 95 (1-6), 233-260.

(7) Dagaut, P.; Reuillon, M.; Cathonnet, M., Experimental-Study of the Oxidation of N-Heptane in a Jet-Stirred Reactor from Low-Temperature to High-Temperature and Pressures up to 40-Atm. Combust. Flame 1995, 101 (1-2), 132-140.

(8) Dagaut, P.; Reuillon, M.; Cathonnet, M.; Presvots, D., Gas-Chromatography and Mass-Spectrometry Identification of Cyclic Ethers Formed from Reference Fuels Combustion. Chromatographia 1995, 40 (3-4), 147-154.

(9) Cullis, C. F.; Fish, A.; Saeed, M.; Trimm, D. L.; Gaydon, A. G., Alkylperoxy radical isomerization and cool flames. Proceedings of the Royal Society of London. Series A. Mathematical and Physical Sciences 1966, 289 (1418), 402-412.

(10) Griffiths, J. F., Reduced kinetic models and their application to practical combustion systems. Prog. Energy Combust. Sci. 1995, 21 (1), 25-107.

(11) Eldeeb, M. A.; Akih-Kumgeh, B., Recent Trends in the Production, Combustion and Modeling of Furan-Based Fuels. . Energies 2018, 11 (3), 512.

(12) Dagaut, P.; Voisin, D.; Cathonnet, M.; McGuinness, M.; Simmie, J. M., The oxidation of ethylene oxide in a jet-stirred reactor and its ignition in shock waves. Combust. Flame 1996, 106 (1-2), $62-68$.

(13) Dagaut, P.; Cathonnet, M.; McGuinness, M.; Simmie, J. M., The ignition of oxetane in shock waves and oxidation in a jet-stirred reactor: An experimental and kinetic modeling study. Combust. Flame 1997, 110 (4), 409-417.

(14) Dagaut, P.; McGuinness, M.; Simmie, J. M.; Cathonnet, M., The ignition and oxidation of tetrahydropyran: Experiments and kinetic modeling. Combust. Sci. Technol. 1997, 129 (1-6), 1-16.

(15) Dagaut, P.; McGuinness, M.; Simmie, J. M.; Cathonnet, M., The ignition and oxidation of tetrahydrofuran. Experiments and kinetic modeling. Combust. Sci. Technol. 1998, 135 (1-6), 3-29.

(16) Dahmen, M.; Marquardt, W., A Novel Group Contribution Method for the Prediction of the Derived Cetane Number of Oxygenated Hydrocarbons. Energy Fuels 2015, 29 (9), 5781-5801.

(17) Molera, M. J.; Couto, A.; Garcia-Dominguez, J. A., Gas phase oxidation of tetrahydrofuran. Int. J. Chem. Kinet. 1988, $20(9), 673-685$.

(18) Vanhove, G.; Yu, Y.; Boumehdi, M. A.; Frottier, O.; Herbinet, O.; Glaude, P.-A.; Battin-Leclerc, F., Experimental Study of Tetrahydrofuran Oxidation and Ignition in Low-Temperature Conditions. Energy Fuels 2015, 29 (9), 6118-6125.

(19) Uygun, Y.; Ishihara, S.; Olivier, H., A high pressure ignition delay time study of 2-methylfuran and tetrahydrofuran in shock tubes. Combust. Flame 2014, 161 (10), 2519-2530.

(20) Antonov, I. O.; Zádor, J.; Rotavera, B.; Papajak, E.; Osborn, D. L.; Taatjes, C. A.; Sheps, L., Pressure-Dependent Competition among Reaction Pathways from First- and Second-O2 Additions in the Low-Temperature Oxidation of Tetrahydrofuran. The Journal of Physical Chemistry A 2016, 120 (33), 6582-6595.

(21) Wu, Y. T.; Xu, N.; Yang, M.; Liu, Y.; Tang, C. L.; Huang, Z. H., Ignition delay time measurement and kinetic modeling of furan, and comparative studies of 2,3-dihydrofuran and tetrahydrofuran at low to intermediate temperatures by using a rapid compression machine. Combust. Flame 2020, 213, 226-236.

(22) Fenard, Y.; Gil, A.; Vanhove, G.; Carstensen, H.-H.; Van Geem, K. M.; Westmoreland, P. R.; Herbinet, O.; BattinLeclerc, F., A model of tetrahydrofuran low-temperature oxidation based on theoretically calculated rate constants. Combust. Flame 2018, 191, 252-269.

(23) Sudholt, A.; Cai, L.; Heyne, J.; Haas, F. M.; Pitsch, H.; Dryer, F. L., Ignition characteristics of a bio-derived class of saturated and unsaturated furans for engine applications. Proc. Combust. Inst. 2015, 35 (3), 2957-2965.

(24) Hansen, N.; Moshammer, K.; Jasper, A. W., Isomer-Selective Detection of KetoHydroperoxides in the LowTemperature Oxidation of Tetrahydrofuran. J. Phys. Chem. A 2019, 123 (38), 8274-8284. 
(25) Wang, Z.; Popolan-Vaida, D. M.; Chen, B.; Moshammer, K.; Mohamed, S. Y.; Wang, H.; Sioud, S.; Raji, M. A.; Kohse-Höinghaus, K.; Hansen, N.; Dagaut, P.; Leone, S. R.; Sarathy, S. M., Unraveling the structure and chemical mechanisms of highly oxygenated intermediates in oxidation of organic compounds. Proceedings of the National Academy of Sciences 2017, 114 (50), 13102-13107.

(26) Belhadj, N.; Benoit, R.; Dagaut, P.; Lailliau, M.; Serinyel, Z.; Dayma, G.; Khaled, F.; Moreau, B.; Foucher, F., Oxidation of di-n-butyl ether: Experimental characterization of low-temperature products in JSR and RCM. Combust. Flame 2020, 222, 133-144.

(27) Belhadj, N.; Benoit, R.; Dagaut, P.; Lailliau, M.; Serinyel, Z.; Dayma, G., Oxidation of di-n-propyl ether: Characterization of low-temperature products. Proc. Combust. Inst. 2021, 38, https://doi.org/10.1016/j.proci.2020.06.350.

(28) Belhadj, N.; Benoit, R.; Dagaut, P.; Lailliau, M., Experimental characterization of n-heptane low-temperature oxidation products including ketohydroperoxides and highly oxygenated organic molecules (HOMs). Combust. Flame 2020.

(29) Wang, Z. D.; Sarathy, S. M., Third O-2 addition reactions promote the low-temperature auto-ignition of n-alkanes. Combust. Flame 2016, 165, 364-372.

(30) Bianchi, F.; Kurtén, T.; Riva, M.; Mohr, C.; Rissanen, M. P.; Roldin, P.; Berndt, T.; Crounse, J. D.; Wennberg, P. O.; Mentel, T. F.; Wildt, J.; Junninen, H.; Jokinen, T.; Kulmala, M.; Worsnop, D. R.; Thornton, J. A.; Donahue, N.; Kjaergaard, H. G.; Ehn, M., Highly Oxygenated Organic Molecules (HOM) from Gas-Phase Autoxidation Involving Peroxy Radicals: A Key Contributor to Atmospheric Aerosol. Chemical Reviews 2019, 119 (6), 3472-3509.

(31) Perrin, O.; Heiss, A.; Sahetchian, K.; Kerhoas, L.; Einhorn, J., Determination of the isomerization rate constant $\mathrm{HOCH} 2 \mathrm{CH} 2 \mathrm{CH} 2 \mathrm{CH}(\mathrm{OO}$ center dot $) \mathrm{CH} 3->(\mathrm{HOCHCH} 2 \mathrm{CH} 2 \mathrm{CH})-\mathrm{H}$-center $\operatorname{dot}(\mathrm{OOH}) \mathrm{CH} 3$. Importance of intramolecular hydroperoxy isomerization in tropospheric chemistry. Int. J. Chem. Kinet. 1998, 30 (12), 875-887.

(32) Blin-Simiand, N.; Jorand, F.; Keller, K.; Fiderer, M.; Sahetchian, K., Ketohydroperoxides and ignition delay in internal combustion engines. Combust. Flame 1998, 112, 278-282.

(33) Heiss, A.; Sahetchian, K., Isomerization reactions of the n-C4H9O and n-OOC4H8OH radicals in oxygen. Int. J. Chem. Kinet. 1996, 28 (7), 531-544.

(34) Sahetchian, K.; Champoussin, J. C.; Brun, M.; Levy, N.; Blin-Simiand, N.; Aligrot, C.; Jorand, F.; Socoliuc, M.; Heiss, A.; Guerassi, N., Experimental study and modeling of dodecane ignition in a diesel engine. Combust. Flame 1995, 103 (3), 207-220.

(35) Zinbo, M.; Jensen, R. K.; Korcek, S., Gas-liquid-chromatography of oxygenated compounds related to autoxidation of n-hexadecane. Anal. Lett. 1977, 10 (2), 119-132.

(36) Jensen, R. K.; Korcek, S.; Mahoney, L. R.; Zinbo, M., Liquid-phase autoxidation of organic-compounds at elevatedtemperatures .1. stirred flow reactor technique and analysis of primary products from normal-hexadecane autoxidation at 120-degrees-C 180-degrees-C. J. Am. Chem. Soc. 1979, 101 (25), 7574-7584.

(37) Jensen, R. K.; Korcek, S.; Mahoney, L. R.; Zinbo, M., Liquid-phase autoxidation of organic-compounds at elevatedtemperatures .2. Kinetics and mechanisms of the formation of cleavage products in normal-hexadecane autoxidation. $J$. Am. Chem. Soc. 1981, 103 (7), 1742-1749.

(38) Jensen, R. K.; Zinbo, M.; Korcek, S., HPLC determination of hydroperoxidic products formed in the autoxidation of normal-hexadecane at elevated-temperatures. J. Chromatogr. Sci. 1983, 21 (9), 394-397.

(39) Jensen, R. K.; Korcek, S.; Zinbo, M., Formation, isomerization, and cyclization reactions of hydroperoxyalkyl radicals in hexadecane autoxidation at 160-190-degrees-C. J. Am. Chem. Soc. 1992, 114 (20), 7742-7748.

(40) Moshammer, K.; Jasper, A. W.; Popolan-Vaida, D. M.; Lucassen, A.; Dievart, P.; Selim, H.; Eskola, A. J.; Taatjes, C. A.; Leone, S. R.; Sarathy, S. M.; Ju, Y. G.; Dagaut, P.; Kohse-Hoinghaus, K.; Hansen, N., Detection and Identification of the KetoHydroperoxide (HOOCH2OCHO) and Other Intermediates during Low-Temperature Oxidation of Dimethyl Ether. $J$. Phys. Chem. A 2015, 119 (28), 7361-7374.

(41) Moshammer, K.; Jasper, A. W.; Popolan-Vaida, D. M.; Wang, Z. D.; Shankar, V. S. B.; Ruwe, L.; Taatjes, C. A.; Dagaut, P.; Hansen, N., Quantification of the KetoHydroperoxide (HOOCH2OCHO) and Other Elusive Intermediates during Low-Temperature Oxidation of Dimethyl Ether. J. Phys. Chem. A 2016, 120 (40), 7890-7901.

(42) Dagaut, P.; Belhadj, N.; Benoit, R.; Dayma, G.; Lailliau, M.; Serinyel, Z., Ketohydroperoxides and Korcek mechanism identified during the oxidation of dipropyl ether in a JSR by high-resolution mass spectrometry. In MCS11 11th Mediterranean Combustion Symposium https://hal.archives-ouvertes.fr/hal-02137413, Tenerife, Spain, 2019.

(43) Dagaut, P.; Cathonnet, M.; Rouan, J. P.; Foulatier, R.; Quilgars, A.; Boettner, J. C.; Gaillard, F.; James, H., A JetStirred Reactor for Kinetic-Studies of Homogeneous Gas-Phase Reactions at Pressures up to 10-Atmospheres ( 1 MPa). Journal of Physics E-Scientific Instruments 1986, 19 (3), 207-209.

(44) Dagaut, P.; Cathonnet, M.; Boettner, J. C.; Gaillard, F., Kinetic Modeling of Propane Oxidation. Combust. Sci. Technol. 1987, 56 (1-3), 23-63.

(45) El Bakali, A.; Dagaut, P.; Pillier, L.; Desgroux, P.; Pauwels, J. F.; Rida, A.; Meunier, P., Experimental and modeling study of the oxidation of natural gas in a premixed flame, shock tube, and jet-stirred reactor. Combust. Flame 2004, 137 (12), 109-128.

(46) Le Cong, T.; Dagaut, P., Experimental and Detailed Modeling Study of the Effect of Water Vapor on the Kinetics of Combustion of Hydrogen and Natural Gas, Impact on NOx. Energy Fuels 2009, 23 (1), 725-734.

(47) Dagaut, P.; Cathonnet, M.; Boettner, J. C.; Gaillard, F., Kinetic modeling of ethylene oxidation. Combust. Flame 1988, 71 (3), 295-312.

(48) Sivaramakrishnan, R.; Brezinsky, K.; Dayma, G.; Dagaut, P., High pressure effects on the mutual sensitization of the oxidation of NO and CH4-C2H6 blends. Phys. Chem. Chem. Phys. 2007, 9 (31), 4230-4244. 
(49) Thion, S.; Togbe, C.; Serinyel, Z.; Dayma, G.; Dagaut, P., A chemical kinetic study of the oxidation of dibutyl-ether in a jet-stirred reactor. Combust. Flame 2017, 185, 4-15.

(50) Nanita, S. C.; Kaldon, L. G., Emerging flow injection mass spectrometry methods for high-throughput quantitative analysis. Anal. Bioanal. Chem. 2016, 408 (1), 23-33.

(51) Ashcroft, A. E., Ionization Methods in Organic Mass Spectrometry. The Royal Society of Chemistry: London, UK, 1997.

(52) Harata, K.; Kitagawa, S.; Iiguni, Y.; Ohtani, H., Identification of polymer species in a complex mixture by pyrolysisgas chromatography-atmospheric pressure chemical ionization-high resolution time-of-flight mass spectrometry as a basis for environmental microplastic analysis. J. Anal. Appl. Pyrolysis 2020, 148, 104828.

(53) Lafleur, A. L.; Taghizadeh, K.; Howard, J. B.; Anacleto, J. F.; Quilliam, M. A., Characterization of flame-generated $\mathrm{C} 10$ to $\mathrm{C} 160$ polycyclic aromatic hydrocarbons by atmospheric-pressure chemical ionization mass spectrometry with liquid introduction via heated nebulizer interface. J. Am. Soc. Mass Spectrom. 1996, 7 (3), 276-286.

(54) Kostyukevich, Y.; Acter, T.; Zherebker, A.; Ahmed, A.; Kim, S.; Nikolaev, E., Hydrogen/deuterium exchange in mass spectrometry. Mass Spectrom. Rev. 2018, 37 (6), 811-853.

(55) Glarborg, P.; Kee, R. J.; Grcar, J. F.; Miller, J. A. PSR: A FORTRAN program for modeling well-stirred reactors.; SAND86-8209; Sandia National Laboratories: Livermore, CA, 1986.

(56) Kee, R. J.; Rupley, F. M.; Miller, J. A. CHEMKIN-II: A Fortran Chemical Kinetics Package for the Analysis of GasPhase Chemical Kinetics.; SAND89-8009; Sandia National Laboratories: Livermore, CA, 1989.

(57) Wang, Z. D.; Chen, B. J.; Moshammer, K.; Popolan-Vaida, D. M.; Sioud, S.; Shankar, V. S. B.; Vuilleumier, D.; Tao, T.; Ruwe, L.; Brauer, E.; Hansen, N.; Dagaut, P.; Kohse-Hoinghaus, K.; Raji, M. A.; Sarathy, S. M., n-Heptane cool flame chemistry: Unraveling intermediate species measured in a stirred reactor and motored engine. Combust. Flame 2018, 187, 199-216.

(58) Antonov, I. O.; Zador, J.; Rotavera, B.; Papajak, E.; Osborn, D. L.; Taatjes, C. A.; Sheps, L., Pressure-Dependent Competition among Reaction Pathways from First- and Second-O-2 Additions in the Low-Temperature Oxidation of Tetrahydrofuran. J. Phys. Chem. A 2016, 120 (33), 6582-6595.

(59) Sheps, L. Measurements of Key Chemical Intermediates in Low-T Combustion; SAND2016-10565C; Sandia National Laboratories, Livermore, CA: October 18, 2016, 2016.

(60) Wu, S.; Tay, K. L.; Yu, W.; Lin, Q.; Li, H.; Zhao, F.; Yang, W., Development of a highly compact and robust chemical reaction mechanism for the oxidation of tetrahydrofurans under engine relevant conditions. Fuel 2020, $276,118034$.

(61) Antonov, I.; Sheps, L., Characterization of reaction pathways in low temperature oxidation of tetrahydrofuran with multiplexed photoionization mass spectrometry technique. In 70th International Symposium on Molecular Spectroscopy, University of Illinois at Urbana-Champaign, 2015; pp 1-1.

(62) Blin-Simiand, N.; Jorand, F.; Sahetchian, K.; Brun, M.; Kerhoas, L.; Malosse, C.; Einhorn, J., Hydroperoxides with zero, one, two or more carbonyl groups formed during the oxidation of n-dodecane. Combust. Flame 2001, 126 (1), $1524-$ 1532 .

(63) Pelucchi, M.; Bissoli, M.; Cavallotti, C.; Cuoci, A.; Faravelli, T.; Frassoldati, A.; Ranzi, E.; Stagni, A., Improved Kinetic Model of the Low-Temperature Oxidation of n-Heptane. Energy Fuels 2014, 28 (11), 7178-7193.

(64) West, R. H.; Goldsmith, C. F., The impact of roaming radicals on the combustion properties of transportation fuels. Combust. Flame 2018, 194, 387-395.

(65) Eskola, A. J.; Antonov, I. O.; Sheps, L.; Savee, J. D.; Osborn, D. L.; Taatjes, C. A., Time-resolved measurements of product formation in the low-temperature $(550-675 \mathrm{~K})$ oxidation of neopentane: a probe to investigate chain-branching mechanism. Phys. Chem. Chem. Phys. 2017, 21, 13731-13745.

(66) Battin-Leclerc, F.; Bourgalais, J.; Gouid, Z.; Herbinet, O.; Garcia, G.; Arnoux, P.; Wang, Z.; Tran, L.-S.; Vanhove, G.; Nahon, L.; Hochlaf, M., Chemistry deriving from OOQOOH radicals in alkane low-temperature oxidation: A first combined theoretical and electron-ion coincidence mass spectrometry study. Proc. Combust. Inst. 2021, https://doi.org/10.1016/j.proci.2020.06.159. 